Vol. 80B, No. 1, January-March 1976

\title{
Plane-Wave Scattering-Matrix Theory of Antennas and Antenna-Antenna Interactions: Formulation and Applications
}

\author{
David M. Kerns \\ Institute for Basic Standards, National Bureau of Standards, Boulder, Colorado 80302
}

(September 29, 1975)

\begin{abstract}
In recent years a considerable amount of theoretical, experimental, and computational work in the development and application of techniques for accurate measurement of microwave antennas has been successfully completed at the National Bureau of Standards (and work is continuing). This paper presents and extends the basic plane-wave scattering-matrix formalism and presents new generalized or adjoint reciprocity relations for antennas. The PWSM formalism is eminently suitable for the formulation and solution of problems involving interactions at arbitrary distances and for the expression of conventional asymptotic quantities, such as gain, effective area, and polarization. It has in particular enabled derivation of two new techniques that permit accurate, "probe-corrected" antenna measurements at greatly reduced distances: (1) by deconvolution of transverse scanning data, taken with $\mathrm{d} \ll d_{R}$ (where $d_{R} \equiv a^{2} / 2 \lambda$ ) and (2) by extrapolation of received signal observed as a function of distance $d$, with $\mathrm{d} \sim d_{R}$. These techniques basically determine the scalar product, $\mathrm{C}$, of two vectors characteristic respectively of the transmitting and the receiving antennas. Formulas for utilization of $C$-data, taking full account of polarization characteristics and not requiring reciprocal antennas, are given for (a) one-unknown-antenna, (b) generalized two-identical-antenna, and (c) generalized three-antenna measurement techniques.
\end{abstract}

Key words: Antenna-antenna interactions; antenna measurements; antenna theory; scattering-matrix theory of antennas.

\section{Contents}

Some conventions and notation used consistently

Introduction

1. Plane-wave scattering-matrix for antennas and scatterers

1.1. Representation of fields on $S_{0}$, definition of $a_{0}$ and $b_{0}$

1.2. Representation of fields in space; definition of $a_{0}(m, \mathbf{K})$ and $b a(m, \mathbf{K})--$

1.3. Definition of scattering matrices for antennas and scatterers $b_{q}(m, \mathbf{K})$

1.4. Power expressions

1.5. Reciprocal relations - a summary

1.6. Power gain, effective area, and polarization indices -23

2. Scattering-matrix analysis of coupled antennas;general solution for system 2-port _ $\ldots$

3. Determination of coupling-product values _ _

3.1. Deconvolution of transverse scanning data; application of sampling theorem . . .

3.2. Received signal as a function of distance and the extrapolation technique $\ldots \ldots \ldots$

4. Utilization of coupling-product data _ _

4.1. One unknown antenna (transmitting or receiving)

4.2. Generalized two-identical antenna techniques _ $\ldots 35$

4.3. Generalized three-antenna techniques _

5. Appendices _...

5.1. Appendix A: Reciprocity theorems

5.2. Appendix B: Dissipative characteristics of media; comparison for mutually adjoint media $\ldots \ldots \ldots 7$

5.3. Appendix C: Two-dimensional, spatial sampling or interpolation theorem _ _ _ _ _ _ _ _ _ _ 48

6. References _. .

\section{Some Conventions and Notation Used Consistently}

(a) Conventions

1. Complex numbers are sometimes called phasors; they are not called vectors. 
2. Bold-face symbols denote vectors or dyadics defined in "ordinary" space or in wavenumber space. Components may be complex numbers.

3. Scalar and vector products of two vectors are denoted by $\mathbf{A} \cdot \mathbf{B}$ and $\mathbf{A} \times \mathbf{B}$, respectively. The scalar product of three vectors taken in the cyclic order $\mathbf{A}, \mathbf{B}, \mathbf{C}$ is denoted by [ABC].

4. A superposed bar denotes the complex conjugate.

5. The magnitude of a complex number $z$ is denoted by $|z|$.

6. The squared magnitude of a vector $\mathbf{V}$ is defined by $\mathbf{V} \cdot \overline{\mathbf{V}}$ and denoted by $|\mathbf{V}|^{2}$.

7. The "square" of a vector $\mathbf{V}$ is defined by $\mathbf{V} \cdot \mathbf{V}$ and denoted by $V^{2}$. Example: $k^{2}=\omega^{2} \mu \epsilon$ (see the list of symbols following).

8. "Transverse" means perpendicular to the $z$ axis unless otherwise indicated.

9. "On-axis" refers to the $z$ axis of coordinates, not to an axis possibly suggested by antenna geometry.

10. The (suppressed) time dependence is $\exp (-i \omega t)$.

(b) Roman Letters

$\mathbf{a}_{q}$ : Complete vectorial spectrum for $\mathbf{E}$ of incident field (p. 14).

$\mathbf{A}_{q}$ : Transverse part of $\mathbf{a}_{q}$ (p. 15).

$a_{0}$ : Incident wave-amplitude in antenna feed transmission line or waveguide (p. 9).

$\mathbf{b}_{q}$ : Complete vectorial spectrum for $\mathbf{E}$ of scattered or radiated field (p. 14).

$\mathbf{B}_{q}$ : Transverse part of $\mathbf{b}_{q}$ (p. 15).

$b_{0}$ : Emergent wave-amplitude in antenna feed transmission line or waveguide.

$d \mathbf{K}$ : Symbolizes surface element in double integrals in $k_{x}, k_{y}$ space.

$d \mathbf{R}$ : Symbolizes surface element in double integrals in $x, y$ space.

E: "Electric field" (complex representation).

$\mathbf{E}_{t}:$ Transverse part of $\mathbf{E}$.

$\mathbf{e}_{x}, \mathbf{e}_{y}, \mathbf{e}_{z}$ : Fixed, orthogonal, right-handed system of unit vectors.

$\mathbf{e}_{\|}, \mathbf{e}_{\perp}, \mathbf{e}_{k}$ : Orthogonal, right-handed system of unit vectors tied to $\mathbf{k}$ ( p. 13).

$G_{q}(\mathbf{K})$ : Power-gain function evaluated in the direction of $\mathbf{k} ; q=1$ or 2 implies $\mathbf{k}^{+} \mathbf{k}^{+}$or $\mathbf{k}^{-}$, respectively (p. 23).

H: "Magnetic field" (complex representation).

$\mathbf{H}_{t}:$ Transverse part of $\mathbf{H}$.

$i: \sqrt{-1}$.

k: Propagation vector; components $k_{x}, k_{y}, k_{z}$.

$\mathbf{k}^{ \pm}$: Propagation vector with $\mathrm{z}$-component equal to $\pm \gamma$.

$\mathbf{K}$ : Transverse part of $\mathbf{k}$ ( $\mathbf{K}$ is chosen real in this work).

$k: \quad \sqrt{\mathbf{k} \cdot \mathbf{k}}=\omega \sqrt{\mu \epsilon}($ a real quantity in this work).

$K: \sqrt{\mathbf{K} \cdot \mathbf{K}}$

$m, n$ : Index taking on values 1,2 and indicating association with unit vectors $\boldsymbol{\kappa}_{1}, \boldsymbol{\kappa}_{2}(\mathrm{p} . \quad$ ).

$p, q$ : Index taking on values 1,2 and indicating association with regions to the "right" and to the "left" of an antenna or scatterer (p. 12).

r: Position vector (a real vector); components $x, y, z$.

$\mathbf{R}$ : Transverse part of $\mathbf{r}$.

$r$ : Magnitude of $\mathbf{r}$.

$R$ : Magnitude of $\mathbf{R}$.

$\mathbf{S}_{0 q}:$ Transverse vectorial receiving characteristic (p. 17).

$\mathbf{s}_{0 q}$ : Complementary receiving characteristic ( p. 25).

$\mathbf{S}_{q 0}$ : Transverse vectorial transmitting characteristic (p. 16).

$\mathbf{s}_{q 0}$ : Complete transmitting characteristic (p. 23).

$w_{q}(\mathbf{K})$ : Polarization index for incident plane-waves (p. 25).

$Y_{0}: \sqrt{\epsilon / \mu}$, wave admittance for simple plane-waves in medium with parameters $\epsilon, \mu$.

(c) Greek Letters

$\gamma: \quad \sqrt{k^{2}-K^{2}}$, taken positive when $K<k$, positive imaginary when $K>k ; k_{z}= \pm \gamma($ p. $)$. $\delta\left(k_{x}\right)$ : Dirac delta "function." 


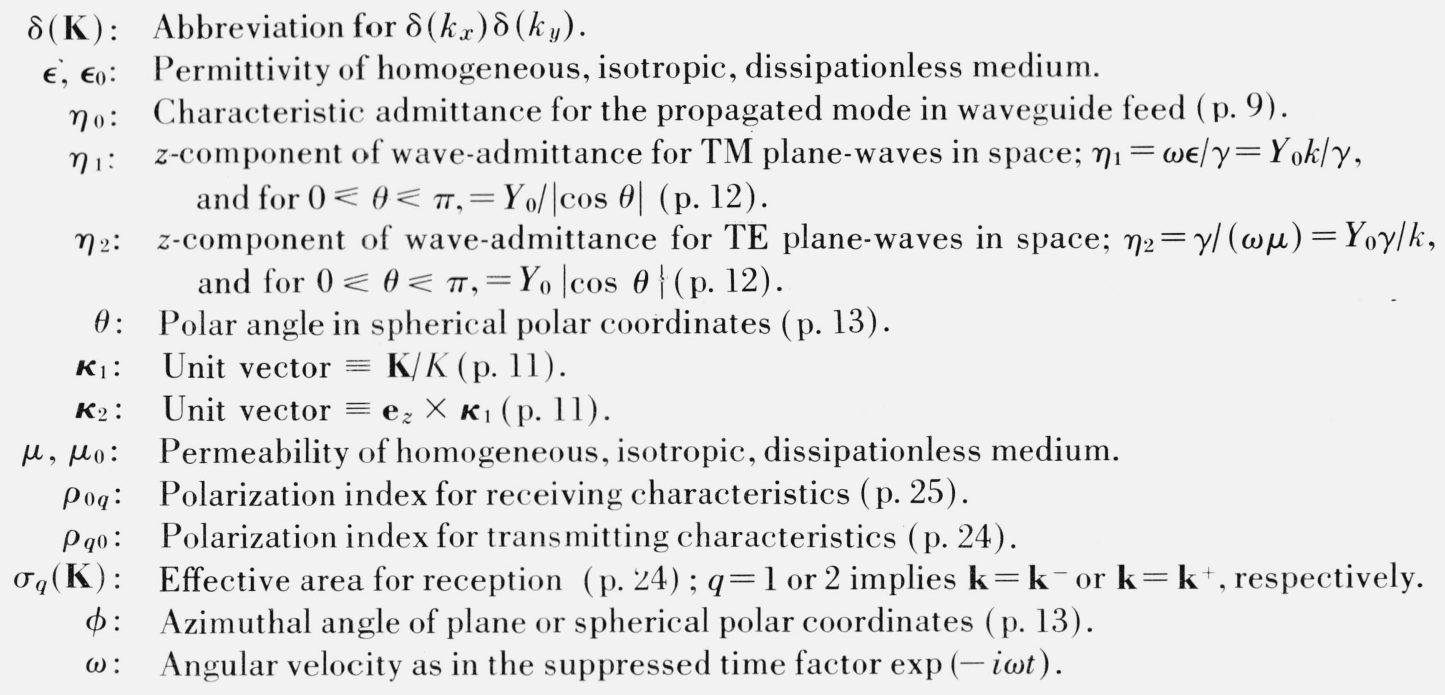

\section{Introduction}

In section 1 we give, quite thoroughly as far as definitions and notation are concerned, a formulation of the plane-wave scattering-matrix for antennas and scatterers. ${ }^{1}$ In previous publications $[1,2],{ }^{2}$ only a "one-side" matrix description of antennas, using one spatial reference plane, was given explicitly. To provide a more complete exposition, we give here the formulation of the complete scattering matrix obtained by enclosing the antenna between two planes and considering incident and emergent plane waves on both planes.

The plane-wave scattering matrix is put forth as a good basis for advanced antenna theory, especially such theory related to antenna measurement techniques. While the results given may be persuasive, numerous analytical examples and applications which illustrate and extend the theory are available-and are not included. These are intended for a more comprehensive publication. Apart from appendices A and B, relatively little theory is developed in this paper. However, logical interrelations are indicated, and more detail is given when it is a question of relating new concepts to more familiar concepts and to practicable antenna measurement techniques.

An "acoustics translation" of much of the present work has been published [3]. Because of the relative simplicity of the acoustic wave-fields involved, this may be found helpful in illuminating the electromagnetic case.

The scattering matrix formulation properly includes basic expressions for power transfer and for reciprocity.

The reciprocity relations are stated in a generalized form, using the concept of mutually adjoint antennas. The concept of generalized reciprocity is not in itself new $[4,5]$, but apparently it has not previously been formulated for antennas and scatterers (see, however, remark following eq. (1.6-21)). The generalized or adjoint reciprocity relations have found substantial application in research establishing the foundations of the extrapolation technique [6, 7]. They are used in this paper in the formulation of possible new antenna measurement techniques, predicated upon the physical realization of mutually adjoint antennas.

In section 2 the plane-wave scattering matrix approach is used to obtain a complete and general solution to the problem of coupled antennas. In spite of their formal appearance, the general results obtained represent the heart of the present theory, and provide a fruitful and reliable basis for additional results (including those reported here). Two of these results are the deconvolution and extrapolation techniques, labeled (1) and (2) in the Abstract and outlined in section 3. These techni-

${ }^{1}$ Since an antenna is in general a scattering object, and a passive antenna externally is merely a scattering object, the consideration of scattering is included in the full consideration of antennas and need not always be mentioned explicitly.

${ }^{2}$ Figures in brackets indicate the literature references at the end of this paper. 
ques basically determine values of the scalar product, called the coupling product, of two two-component vectors characteristic of the two antennas involved. (This statement also applies to the convetional far-field antenna measurement methods, provided due attention is paid to polarization characteristics.)

Utilization of coupling-product data is discussed as a separate topic. Here the analytical problem is primarily geometric and algebraic, with the exact form depending upon what is considered known a priori and what information is sought. Three classes of antenna measurement situations [labeled (a), (b), and (c) in the Abstract] are discussed in section 4. The order of listing and discussion is roughly that of decreasing a priori information and increasing complexity.

An increasing body of experimental results involving various combinations of the several techniques identified above may be found in the literature [8-11, 54]. Moreover, the error analysis required for determination of accuracy in concrete measurement situations is approaching completion (Kanda [12], Yaghjian [13], Newell [14]).

The combination of the techniques labeled (1) and (a) amounts to a technique for correction of near-field antenna measurements made with an arbitrary but known measuring antenna. The ability to obtain the true radiated spectrum of an unknown antenna, fully corrected for the effects of the measuring antenna, incidentally implies the ability to obtain corresponding true values of $\mathbf{E}$ and $\mathbf{H}$ in the near field, similarly fully corrected. Frequently, and in particular in the following paragraphs, the measuring antenna will be referred to as a "probe."

The general subject of determination of far-field antenna patterns from near field data is surveyed and an extensive bibliography is given in a recent paper by Johnson et al. [15]. This paper should be consulted for an overview of the subject. Here we mention specifically only certain earlier work in which the "probe-correction problem" was considered or which represented steps leading to the eventual simple, rigorous, and general deconvolution solution of the problem.

Woonton, in 1953 [16], obtained an integral expression for the near-field response of a linear (=thin wire) antenna and discussed probe effects qualitatively. Woonton stated that the problem had not been critically discussed previously. Dayhoff (1956) [17], using scalar waves, plane-wave spectrum analysis, and reciprocity, introduced a version of the very important transmission integral. (Dayhoff used the transmission integral to obtain an approximate solution of the diffraction correction problem in microwave interferometry. A rigorous and more general version of this solution was presented by Kerns in 1957 [8].) Brown [19] (1958), using plane-wave spectrum analysis and reciprocity [20], obtained a version of the transmission integral and used it to give an approximate analysis of probe effects, assuming simple, known data for both antennas involved. More work along this line (limited to two-dimensions) was done by Jull [21, 22]. In 1961 Brown and Jull [23] gave a rigorous and general solution to the probe correction problem for the two-dimensional case using cylindrical wave functions. The use of two-dimensional solutions in certain three-dimensional problems has been suggested, and was studied experimentally by Martin [24], but is not valid for any three-dimensional problems. The proper extension to three dimensions in spherical or cylindrical coordinates is far from trivial. See Jensen [25], Leach and Paris [26], and W acker [27].

The key to the present solution to the probe-correction problem is the use of plane-wave analysis and rectangular coordinates and the recognition that planar scanning would permit solution of the integral equation presented by the transmission integral by Fourier inversion ${ }^{3}$ (better called deconvolution in the existing context). This solution was presented in 1963 by Kerns [28], and again, including application of a two-dimensional, spatial sampling theorem, in 1967, at a University of Colorado Advanced Electromagnetic Theory Summer Course. Archival publication, accompanied by substantial experimental application and verification by Baird et al. [8], was accomplished in 1970 [2]. The transmission integral used, though similar to that derived by Brown, was actually obtained by methods used in Kerns and Dayhoff [1], where it was derived without recourse to reciprocity and was explicitly recognized as the first term in an infinite series of interaction terms. (See the remarks at the end of appendix A, below.)

${ }^{3}$ These matters are adequately discussed later in this paper. 
The Kerns and Dayhoff paper, not originally considered to be in the domain of antenna theory by its authors, has served as an important base for much of the material embodied in the present and related papers and in other work as yet unpublished.

\section{Plane-Wave Scattering-Matrix for Antennas and Scatterers}

\subsection{Representation of Fields on $S_{0}$, Definition of $a_{0}$ and $b_{0}$}

Let us consider the antenna system shown schematically in figure 1. We choose a (mathematical) terminal surface $S_{0}$ in the waveguide feed and define a supplementary surface $S_{a}$, such that $S_{a}+S_{0}$ forms a closed surface enclosing the source or detector associated with the antenna. The surface $S_{a}$ coincides with shielding, which is required to make the problem well defined (both experimentally and theoretically!). As an important measure of simplicity, we consider only the case of a single waveguide feed, supporting just one propagated mode. We employ conventional phasor wave amplitudes $a_{0}$ and $b_{0}$ for the incident and emergent traveling wave components at $S_{0}$. These wave amplitudes are fully defined by the following four equations.

The tangential (= transverse) components of $\mathbf{E}$ and $\mathbf{H}$ on $S_{0}$ are given by

$$
\begin{aligned}
& \mathbf{E}_{0 t}=\left(a_{0}+b_{0}\right) \mathbf{e}_{0}(\mathbf{r}), \\
& \mathbf{H}_{0 t}=\eta_{0}\left(a_{0}-b_{0}\right) \mathbf{h}_{0}(\mathbf{r}), \quad\left(\mathbf{r} \text { on } S_{0}\right)
\end{aligned}
$$

where $\mathbf{e}_{0}(\mathbf{r})$ and $\mathbf{h}_{0}(\mathbf{r})$ are real basis fields for the mode involved, subject to the impedance normalization

$$
\mathbf{h}_{0}(\mathbf{r})=\eta_{0}^{-1} \eta_{w} \mathbf{n}_{0} \times \mathbf{e}_{0}(\mathbf{r})
$$

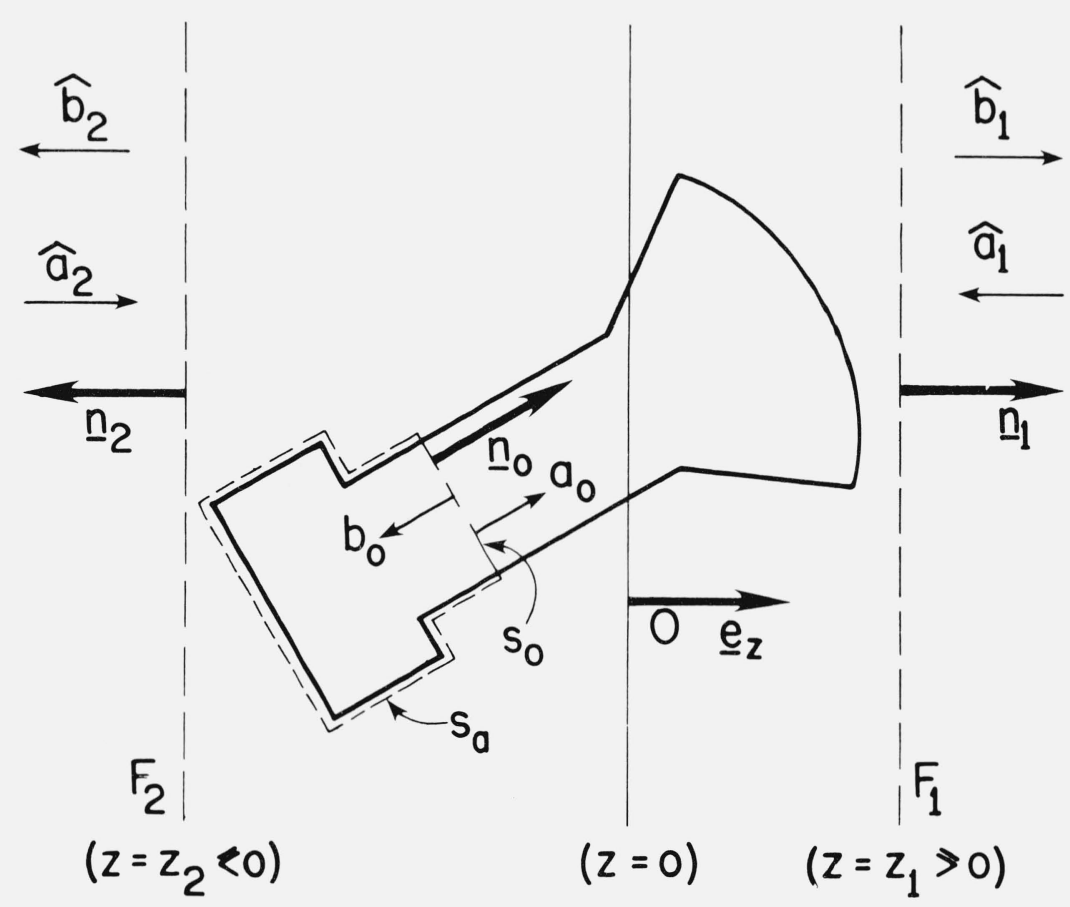

FIGURE 1. Some notation for plane-wave scattering-matrix description of antennas.

Arrows indicate the association of $a$ 's and $b$ 's with incident and emergent waves respectively. Antenna-system representation is symbolic: no particular size. shape, symmetry, type, orientation, or position is implied. In any concrete case, position and orientation of antenna relative to coordinate system must be established. as with the aid of fiducial marks on the antenna. 
and to the power normalization

$$
\int_{S_{0}}\left[\mathbf{e}_{0} \mathbf{h}_{0} \mathbf{n}_{0}\right] d S=1
$$

Here the integrand is the scalar triple product; $\mathbf{n}_{0}$ is the unit normal vector, drawn inward with respect to the antenna; $\eta_{0}$ is the characteristic admittance, and $\eta_{w}$ is the wave admittance for the mode involved. Equation (3) establishes peak-value normalization for $a_{0}$ and $b_{0}$, so that net timeaverage power input to the antenna at $S_{0}$ is given by

$$
P_{0}=\frac{1}{2} \operatorname{Re} \int_{S_{0}} \mathbf{E}_{0 t} \times \overline{\mathbf{H}}_{0 t} \cdot \mathbf{n}_{0} d S=\frac{1}{2} \eta_{0}\left(\left|\boldsymbol{a}_{0}\right|^{2}-\left|b_{0}\right|^{2}\right),
$$

where Re denotes that the real part is to be taken, the superposed bar denotes the complex conjugate, and the vertical bars denote absolute values. Remarks: (a) The impedance normalization shown above is more flexible than that used previously $[1,2]$. It allows one to choose whatever characteristic impedance or admittance is deemed familiar or convenient for purposes of measurement-related calculations. Examples are, for waveguide, $\eta_{0}=1$ or $\eta_{0}=\eta_{w}$; and, for coaxial line, the conventional characteristic admittance $(=2 \pi \sqrt{\epsilon / \mu} / \ln (b / a)$ in conventional notation). (b) Power normalization differs slightly from that used previously: Powers of the factor $(2 \pi)$ have been redistributed. (c) For a detailed discussion of the material in this subsection and for the small but essential amount of microwave network theory needed in measurement-related calculations, see [29] or the especially prepared report [30]. For more elementary discussion see also [31].

\subsection{Representation of Fields in Space; Definition of $a_{q}(m, K)$ and $b_{q}(m, K)$}

We choose a rectangular coordinate system $0 x y z$ (with unit vectors $\mathbf{e}_{x}, \mathbf{e}_{y}$, and $\mathbf{e}_{z}$ ) so that the considered antenna system may be confined entirely to the space between the (mathematical) surfaces $F_{1}$ and $F_{2}$ at $z=z_{1}>0$ and at $z=z_{2} \gtrless 0$, as suggested in figure 1. The electromagnetic fields in the regions to the "right" and to the "left" are to be represented as superpositions of plane-wave solutions of Maxwell's equations. This type of representation is well known (see e.g., [32]), at least for solutions of the scalar Helmholtz equation; an appropriate generalization to the (vector) electromagnetic field, though often shunned, offers no particular difficulty.

The electromagnetic field in the regions under consideration satisfies Maxwell's equations in the form

$$
\nabla \times \mathbf{E}=i \omega \mu \mathbf{H}, \quad \nabla \times \mathbf{H}=-i \omega \epsilon \mathbf{E},
$$

where $\mu, \epsilon$ are constant real scalars representing respectively the permeability and the permittivity of the medium, and $\exp (-i \omega t)$ time dependence is assumed. We derive our basis fields from the general plane wave

$$
\left.\begin{array}{l}
\mathbf{E}=\mathbf{T} \exp (i \mathbf{k} \cdot \mathbf{r}), \\
\mathbf{H}=(\omega \mu)^{-1} \mathbf{k} \times \mathbf{T} \exp (i \mathbf{k} \cdot \mathbf{r}),
\end{array}\right\}
$$

which is a solution of (1) for any propagation vector $\mathbf{k}$ such that $k^{2} \equiv \mathbf{k} \cdot \mathbf{k}=\omega^{2} \mu \epsilon$ and any vector $\mathbf{T}$ (independent of position $\mathbf{r}$ ) satisfying the transversality relation

$$
\mathbf{k} \cdot \mathbf{T}=0 .
$$

In spite of this occurrence of "transversality," in what follows the term "transverse" will mean transverse with respect to the $z$ direction unless otherwise specified.

The propagation vector will be regarded as a function of its transverse components $k_{x}, k_{y}$ (which are chosen real); the $z$-component is thus

$$
k_{z}= \pm \gamma,
$$


where $\gamma^{2}=k^{2}-k_{x}^{2}-k_{y}^{2}$. It will be convenient to denote the transverse part of the propagation vector by $\mathbf{K}$, so that $\mathbf{K}=k_{x} \mathbf{e}_{x}+k_{y} \mathbf{e}_{y}$ and

$$
\gamma=\left(k^{2}-K^{2}\right)^{1 / 2}
$$

Since $k_{x}, k_{y}$ must be allowed to vary independently in the range $(-\infty, \infty)$, real and imaginary values of $\gamma$ will occur. $\gamma$ will be taken positive for $K^{2}<k^{2}$, positive imaginary for $K^{2}>k^{2}$. Superscripts "+" and "-" will be used when it is desired to indicate the choice of sign associated with $k_{z}$. When $\gamma$ is real, the exponentials $\exp \left(i \mathbf{k}^{+} \cdot \mathbf{r}\right)$ and $\exp \left(i \mathbf{k}^{-} \cdot \mathbf{r}\right)$ respectively represent simple plane waves traveling into the $+z$ and $-z$ hemispheres. When $\gamma$ is imaginary, the exponentials represent inhomogeneous plane waves with propagation of phase in the transverse directions and exponential attenuation of amplitude ("evanescence") in the + and $-z$ directions, respectively.

In virtue of the relation $\mathbf{k} \cdot \mathbf{T}=0,(2 \mathrm{a})$ yields just two linearly independent fields, hence just two basis fields, for any given k. The appropriate polarizations for the basis fields are those with the electric vectors parallel or perpendicular to the plane of $\mathbf{k}$ and $\mathbf{e}_{z}$, which is the plane of incidence for a wave incident on any plane $z=$ const. This choice of polarizations yields "transverse magnetic" and "transverse electric" waves; the same choice of polarizations, usually labelled " $E_{\| !}$" and " $E_{\perp} "$, simplifies the derivation of Fresnel's equations in optics or electromagnetic theory.

In order to set up the basis fields in the desired form, we require the transverse unit vectors

$$
\boldsymbol{\kappa}_{1}=\mathbf{K} / K, \quad \boldsymbol{\kappa}_{2}=\mathbf{e}_{z} \times \boldsymbol{\kappa}_{1},
$$

which are respectively in and perpendicular to the plane of $\mathbf{k}$ and $\mathbf{e}_{z}$. This part of the notation is illustrated in figure $2 ; \kappa_{1}$ and $\boldsymbol{\kappa}_{2}$ may be identified as radial and tangential unit vectors, as often

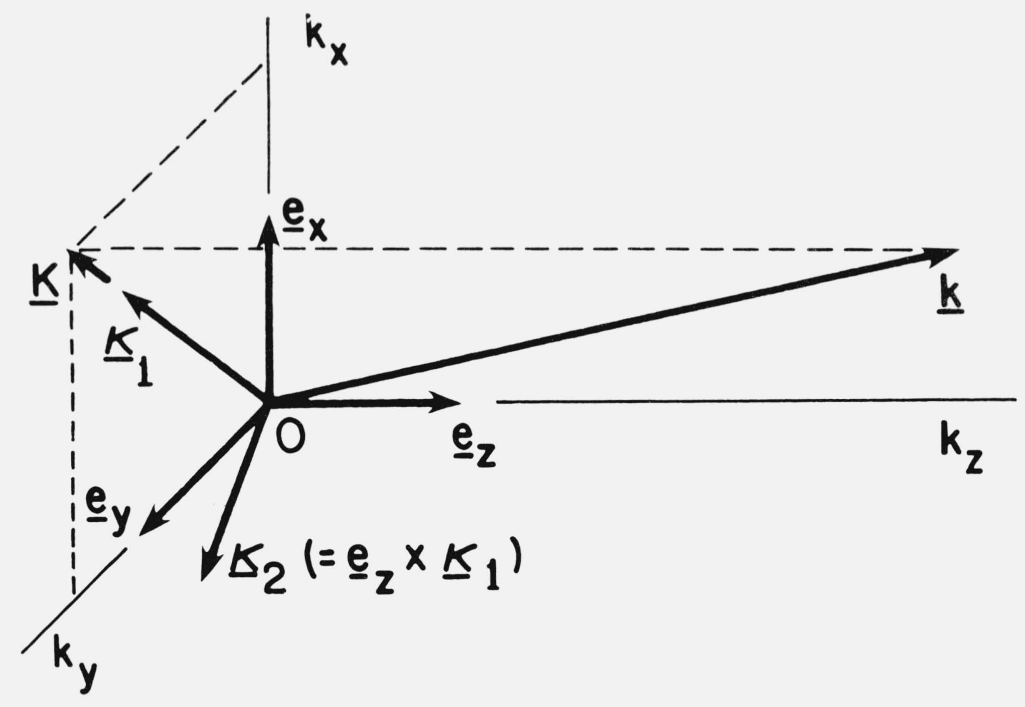

FIGURE 2. Illustrating $\mathbf{k}, \mathbf{K}, \boldsymbol{\kappa}_{1}$, and $\boldsymbol{\kappa}_{2}$.

associated with polar coordinates in a plane. As a temporary abbreviation ${ }^{4}$ we put $u^{ \pm}=\exp \left(i \mathbf{k}^{ \pm} \cdot \mathbf{r}\right) /$ $(2 \pi)$. For the $E_{\|}$(or $\left.T M\right)$ components we put $\mathbf{T}=\boldsymbol{\kappa}_{1} \mp K \gamma^{-1} \mathbf{e}_{z}$ and obtain from (2a)

$$
\left.\begin{array}{l}
\mathbf{E}_{1}^{ \pm}=\left[\boldsymbol{\kappa}_{1} \mp K \gamma^{-1} \mathbf{e}_{z}\right] u^{ \pm}, \\
\mathbf{H}_{1}^{+}= \pm \eta_{1} \mathbf{e}_{z} \times \boldsymbol{\kappa}_{1} u^{ \pm},
\end{array}\right\}
$$

${ }^{4}$ The factor $1 /(2 \pi)$ in the definition of $u$ represents a change in normalization consistent with that noted for $a_{0}$ and $b_{0}$ in the preceding subsection. 
where $\eta_{1}=\omega \epsilon / \gamma$. For the $E_{\perp}$ (or $T E$ ) components we take $\mathbf{T}=\boldsymbol{\kappa}_{2}$ and obtain from (2a)

$$
\left.\begin{array}{l}
\mathbf{E}_{2}^{ \pm}=\boldsymbol{\kappa}_{2} u^{ \pm}, \\
\mathbf{H}_{2}^{ \pm}=\left[ \pm \eta_{2} \mathbf{e}_{z} \times \boldsymbol{\kappa}_{2}+K(\omega \mu)^{-1} \mathbf{e}_{z}\right] u^{ \pm},
\end{array}\right\}
$$

where $\eta_{2}=\gamma /(\omega \mu)$. Among other similarities it may be observed that $\eta_{1}, \eta_{2}$ are wave-admittances that correspond closely to the wave-admittances encountered in the theory of rectangular waveguide. Equations (5) and (6) furnish the desired basis fields; somewhat arbitrarily, we have chosen to make the expressions for transverse $\mathbf{E}$ as simple as possible. The normalization and orthogonality properties of the basis fields are of course implicit in the expressions themselves. (This is an interesting contrast to (1.1-1), where the field patterns are implicit and the normalizations explicit.)

Let us now examine the plane-wave representations of electromagnetic fields in the regions $z \geqslant z_{1}>0$ and $z \gtrless z_{2}<0$. We write

$$
\begin{aligned}
& \mathbf{E}_{q}(\mathbf{r})=\int_{-\infty}^{\infty} \int \sum_{m=1}^{2}\left[b_{q}(m, \mathbf{K}) \mathbf{E}_{m}^{ \pm}(\mathbf{K}, \mathbf{r})+a_{q}(m, \mathbf{K}) \mathbf{E}_{m}^{\mp}(\mathbf{K}, \mathbf{r})\right] d k_{x} d k_{y}, \\
& \mathbf{H}_{q}(\mathbf{r})=\int_{-\infty}^{\infty} \int \sum_{m=1}^{2}\left[b_{q}(m, \mathbf{K}) \mathbf{H}_{m}^{ \pm}(\mathbf{K}, \mathbf{r})+a_{q}(m, \mathbf{K}) \mathbf{H}_{m}^{\mp}(\mathbf{K}, \mathbf{r})\right] d k_{x} d k_{i y} .
\end{aligned}
$$

Here the index $q$ takes on the values 1 and 2 and identifies quantities respectively associated with the regions to the "right" and to the "left" of the system considered; the upper and the lower superscript signs are associated with $q=1$ and $q=2$, respectively; and $b_{q}(m, \mathbf{K})$ and $a_{q}(m, \mathbf{K})$ are scalar spectral-density functions for outgoing and incoming waves, respectively. The electromagnetic fields given by (7) will satisfy Maxwell's equations provided that the necessary differentiations can be taken under the integral signs.

Now, as will be shown in a moment, a knowledge of the transverse components of $\mathbf{E}_{q}$ and $\mathbf{H}_{q}$ (in a single plane, in fact) is sufficient to determine $a_{q}(m, \mathbf{K})$ and $b_{q}(m, \mathbf{K})$; and hence, by (7), the entire electromagnetic field in each of the regions considered. The $z$-components of the fields in (7) are, strictly speaking, redundant. The transverse components are both necessary and sufficient for the expression of normal energy-flux and continuity conditions across a transverse plane. We find, in fact, that the inclusion of $z$-components is sometimes convenient and sometimes not. The following equations illustrate the latter case.

For the transverse components of $\mathbf{E}$ and $\mathbf{H}$ in the regions $z \geqslant z_{1} \geqslant 0$ and $z \leqslant z_{2} \leqslant 0$, we find from (5), (6) and (7) the Fourier integral representations

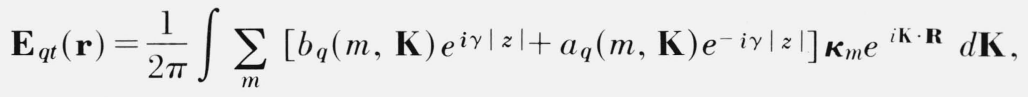

$$
\begin{aligned}
& \mathbf{H}_{q t}(\mathbf{r})=\frac{1}{2 \pi} \int \sum_{m}\left[b_{q}(m, \mathbf{K}) e^{i \gamma|z|} a_{q}(m, \mathbf{K}) e^{-i \gamma|z|}\right] \mathbf{n}_{q} \times \boldsymbol{\kappa}_{m} \eta_{m}(K) e^{i \mathbf{K} \cdot \mathbf{R}} d \mathbf{K},
\end{aligned}
$$

where $\eta_{1}=\omega \epsilon / \gamma, \eta_{2}=\gamma /(\omega \mu)$, and $\mathbf{r}=\mathbf{R}+z \mathbf{e}_{z}$. Here and in subsequent expressions of this type summation over the values 1 and 2 of the polarization index $m$ and integration over the infinite $k_{x}, k_{y}$ plane is to be understood. The role of the index $q$ is as described under (7); the use of $|z|$ and $-|z|$ assures proper phase variation for outgoing and incoming waves, respectively; and $\mathbf{n}_{q}$ is the outward normal unit vector on $F_{q}: \mathbf{n}_{1}=\mathbf{e}_{z}, \mathbf{n}_{2}=-\mathbf{e}_{z}$ (i.e., "outward" means with respect to the slab between the surfaces $F_{1}$ and $\left.F_{2}\right)$. The functions $a_{q}(m, \mathbf{K})$ and $b_{q}(m, \mathbf{K})$ may be regarded as modal terminal variables for the continuous spectrum; a mode is identified by a triplet of values $\left(m, k_{x}, k_{y}\right)$ and a direction (rightward or leftward). 
Explicit expressions for the spectral density functions $b_{q}(m, \mathbf{K})$ and $a_{q}(m, \mathbf{K})$ may be found from the Fourier inversion of the above equations:

$$
\begin{aligned}
& b_{q}(m, \mathbf{K})=\frac{e^{-i \gamma|z|}}{4 \pi} \boldsymbol{\kappa}_{m} \cdot \int\left[\mathbf{E}_{q}(\mathbf{R}, z)+\eta_{m}^{-1} \mathbf{H}_{q}(\mathbf{R}, z) \times \mathbf{n}_{q}\right] e^{-i \mathbf{K} \cdot \mathbf{R}} d \mathbf{R}, \\
& a_{q}(m, \mathbf{K})=\frac{e^{i \gamma|z|}}{4 \pi} \boldsymbol{\kappa}_{m} \cdot \int\left[\mathbf{E}_{q}(\mathbf{R}, z)-\eta_{m}^{-1} \mathbf{H}_{q}(\mathbf{R}, z) \times \mathbf{n}_{q}\right] e^{-i \mathbf{K} \cdot \mathbf{R}} d \mathbf{R} .
\end{aligned}
$$

Here the integrations are to be taken over the entire $x, y$ plane-as required by the Fourier inversion - for any suitable fixed value of $z$ (for $q=1, z \geqslant z_{1}$; for $q=2, z \leqslant z_{2}$ ).

The spectral functions $b_{q}(m, \mathbf{K})$ and $a_{q}(m, \mathbf{K})$ are independent of $z$, although this may not be immediately apparent in (9), and the values of the functions are referred to the plane $z==0$. If desired, the phases and amplitudes could be referred to other reference planes $S_{q}$ at $z=Z_{q}$, say. However, the choice implicitly made, $Z_{1}=Z_{2}=0$, is convenient at least for present purposes.

It is convenient at this point to introduce a number of definitions for future reference when and as needed.

We observe that (7a) can be written

$$
\mathbf{E}_{q}(\mathbf{r})=\frac{1}{2 \pi} \int\left[\mathbf{b}_{q}(\mathbf{K}) e^{i \gamma|z|}+\mathbf{a}_{q}(\mathbf{K}) e^{-i \gamma|z|}\right] e^{i \mathbf{K} \cdot \mathbf{R}} d \mathbf{K}
$$

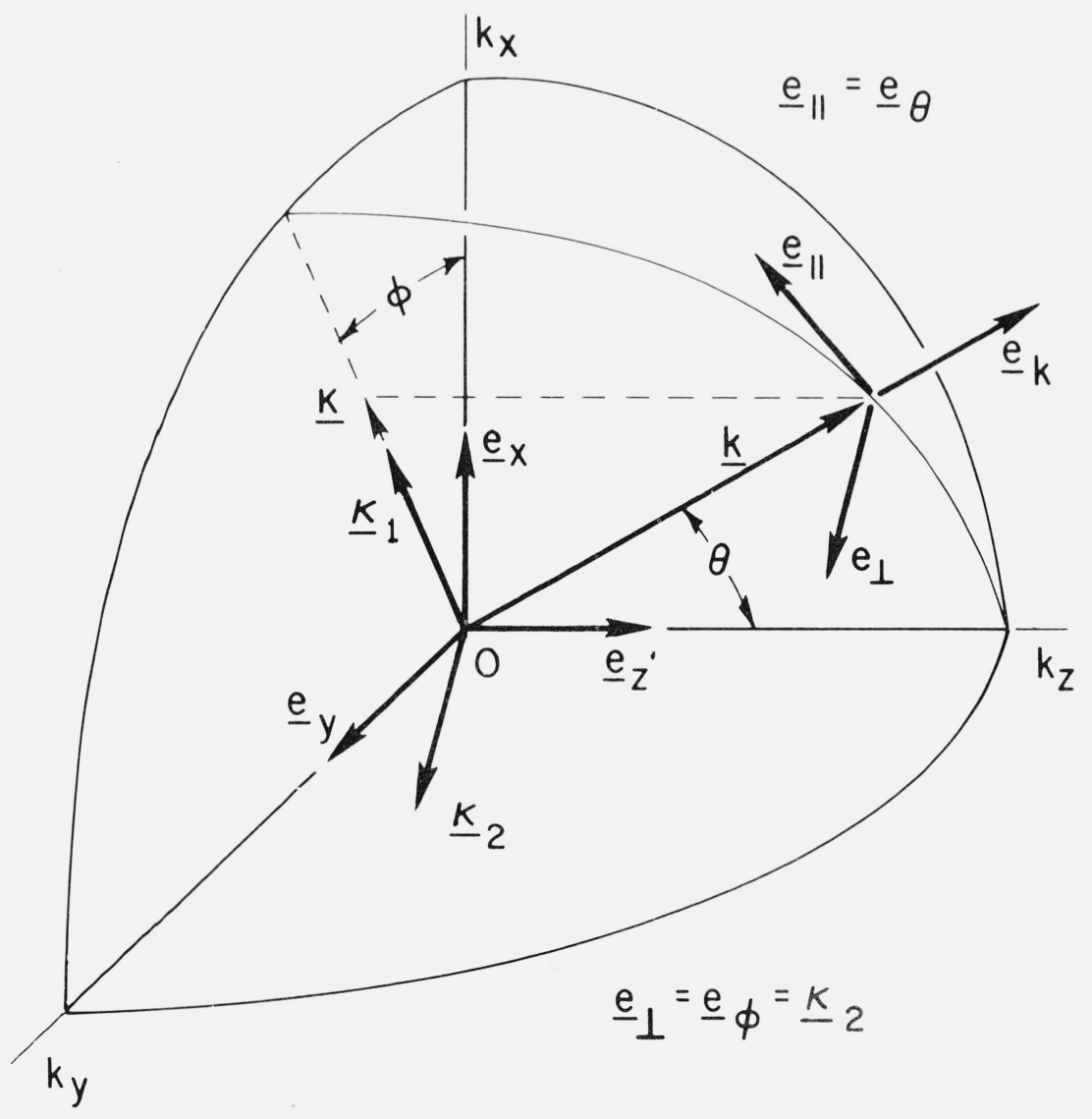

FigURE 3. Unit vectors associated with $\mathbf{k}$ and $\mathbf{K}$ (for real $\mathbf{k})$. See also table 1 . 
TABLE 1. Unit Vectors Associated With $\mathbf{K}$ and $\mathbf{k}$

$\mathbf{K}:\left(k_{x}, k_{y}\right)=(K \cos \phi, K \sin \phi)$
$\boldsymbol{\kappa}_{1}=\mathbf{K} / K=\left(k_{x} \mathbf{e}_{x}+k_{y} \mathbf{e}_{y}\right) / K=\cos \phi \mathbf{e}_{x}+\sin \phi \mathbf{e}_{y}$
$\boldsymbol{\kappa}_{2} \equiv \mathbf{e}_{z} \times \boldsymbol{\kappa}_{1}=\left(-k_{y} \mathbf{e}_{x}+k_{x} \mathbf{e}_{y}\right) / K=-\sin \phi \mathbf{e}_{x}+\cos \phi \mathbf{e}_{y} \quad$
$\left[\boldsymbol{\kappa}_{1} \boldsymbol{\kappa}_{2} \mathbf{e}_{z}\right]=1, \quad \boldsymbol{\kappa}_{m}(-\mathbf{K})=-\boldsymbol{\kappa}_{m}(\mathbf{K})$
$\mathbf{e}_{k} \equiv \mathbf{k} / k=\left(K \kappa_{x}, k_{y}, k_{z}\right)=(k \sin \theta \cos \phi, k \sin \theta \sin \phi, k \cos \theta)$
$\left.\mathbf{e}_{\|} \equiv \boldsymbol{\kappa}_{2} \times \mathbf{e}_{z}\right) / k=\sin \theta \boldsymbol{\kappa}_{1}+\cos \theta \mathbf{e}_{z}$
$\left[k_{z} \boldsymbol{\kappa}_{1}-K \mathbf{e}_{z}\right) / k=\cos \theta \boldsymbol{\kappa}_{1}-\sin \theta \mathbf{e}_{z}$
$\mathbf{e}_{\perp} \equiv \boldsymbol{\kappa}_{2}$
$\mathbf{e}_{\|}(-\mathbf{k})=\mathbf{e}_{\|}(\mathbf{k}), \quad k_{z}= \pm \gamma$

Notes:

(a) $\kappa_{1}$ and $\boldsymbol{\kappa}_{2}$ are respectively "radial" and "tangential" unit vectors associated with the plane polar coordinates for $\mathbf{K}$. These unit vectors are always real.

(b) In the propagating regime, where $\mathbf{e}_{\|}$as well as $\mathbf{e}_{\perp}$ is real, $\mathbf{e}_{\|}$and $\mathbf{e}_{\perp}$ may also be identified as the customary $\theta$ and $\phi$ unit-vectors of the spherical polar coordinates for $\mathbf{k}$. The "middle" forms in the table help show what happens when $K>k$ : $\mathbf{e}_{\|}$and $\mathbf{e}_{k}$ become complex, but remain unit vectors in the sense $\mathbf{e}_{\|} \cdot \mathbf{e}_{\|}=\mathbf{e}_{k} \cdot \mathbf{e}_{k}=1$.

(c) The unit vectors $\boldsymbol{\kappa}_{1}, \boldsymbol{\kappa}_{2}$, and $\mathbf{e}_{\|}$are not defined by the equations in the table at the singular points $K=0$ and $\theta=0, \pi$. For $\mathbf{k}=k \mathbf{e}_{z}$, one may, e.g., determine a consistent set by choosing $\boldsymbol{\kappa}_{1}=\mathbf{e}_{x}$. (cf. use of (4.1-4)).

Here $\mathbf{b}_{q}$ and $\mathbf{a}_{q}$, the "complete vectorial spectra" for the outgoing and incoming plane-wave components of $\mathbf{E}_{q}$, respectively, are given by

$$
\begin{aligned}
& \mathbf{b}_{q}(\mathbf{K})=b_{q}(1, \mathbf{K})\left(\boldsymbol{\kappa}_{1} \mp K \gamma^{-1} \mathbf{e}_{z}\right)+b_{q}(2, \mathbf{K}) \boldsymbol{\kappa}_{2}, \\
& \mathbf{a}_{q}(\mathbf{K})=a_{q}(1, \mathbf{K})\left(\boldsymbol{\kappa}_{1} \pm K \gamma^{-1} \mathbf{e}_{z}\right)+a_{q}(2, \mathbf{K}) \boldsymbol{\kappa}_{2} .
\end{aligned}
$$

(The association of the upper and lower signs with $q=1$ and $q=2$, respectively, is continued from (7).) Alternatively, $\mathbf{b}_{q}$ and $\mathbf{a}_{q}$ may be expressed as follows:

$$
\begin{aligned}
& \mathbf{b}_{q}(\mathbf{K})=b_{q}(1, \mathbf{K})\left[(-)^{q-1} k / \gamma\right] \mathbf{e}_{\|}(\mathbf{k})+b_{q}(2, \mathbf{K}) \mathbf{e}_{\perp}(\mathbf{K}), \\
& \mathbf{a}_{q}(\mathbf{K})=a_{q}(1, \mathbf{K})\left[(-)^{q} k / \gamma\right] \mathbf{e}_{\|}(\mathbf{k})+a_{q}(2, \mathbf{K}) \mathbf{e}_{\perp}(\mathbf{K}),
\end{aligned}
$$

where $\mathbf{e}_{\|}(\mathbf{k})$ is defined as $\boldsymbol{\kappa}_{2} \times \mathbf{k} / k$, and $\mathbf{e}_{\perp}(\mathbf{K})$ is a suggestive alternative notation for $\boldsymbol{\kappa}_{2}$. (The factors in brackets in (12) are introduced as a convenient, explicit way of helping to keep signs straight.) The complete vectorial spectra are of interest to us primarily in the propagating regime, where $\mathbf{e}_{\|}$, as well as $\mathbf{e}_{\perp}$, is real.

All the unit vectors associated with $\mathbf{k}$ and $\mathbf{K}$ are pictured in figure 3 and fully identified and related in table 1.

The complete vectorial spectra must and do satisfy the transversality relations

$$
\mathbf{k}^{ \pm} \cdot \mathbf{b}_{q}=0, \quad \mathbf{k}^{\mp} \cdot \mathbf{a}_{q}=0,
$$

which indeed are expressions of the transversality of the basis fields (5) and (6). 
We next observe that $(8 \mathrm{a})$ can be written

$$
\mathbf{E}_{q t}(\mathbf{r})=\frac{1}{2 \pi} \int\left[\mathbf{B}_{q}(\mathbf{K}) e^{i \gamma|z|}+\mathbf{A}_{q}(\mathbf{K}) e^{-i \gamma|z|}\right] e^{i \mathbf{K} \cdot \mathbf{R}} d \mathbf{K}
$$

where $\mathbf{B}_{q}$ and $\mathbf{A}_{q}$, the "transverse vectorial spectra" for the outgoing and incoming plane-wave components of $\mathbf{E}_{q t}$, respectively, are given by

$$
\begin{aligned}
\mathbf{B}_{q}(\mathbf{K}) & =\sum_{m} b_{q}(m, \mathbf{K}) \boldsymbol{\kappa}_{m} \\
\mathbf{A}_{q}(\mathbf{K}) & =\sum_{m} a_{q}(m, \mathbf{K}) \boldsymbol{\kappa}_{m}
\end{aligned}
$$

Further we note that, given (15), $z$-components can be recovered with the aid of the transversality relations (13) or simply by inspection of (11).

Remarks: (a) One may observe a switch - from scalar spectra and vector waves in (7) and (8) to vector spectra and scalar waves in (10) and (14). Equation (7) remains fundamental. (b) The use of the lower case letters $\mathbf{a}$ and $\mathbf{b}$ to denote complete spectra and the use of the capitals $\mathbf{A}$ and $\mathbf{B}$ to denote the corresponding transverse parts should be noted. This parallels our use of the letters $\mathbf{r}, \mathbf{k}$ and $\mathbf{R}, \mathbf{K}$.

The representations (7) and (8) afford a similar and essential resolution of any electromagnetic field into two major parts: that represented by incoming waves and that represented by outgoing waves. The role of the former part is identified variously by terms such as incident, exciting, primary, or incoming; the latter, by terms such as induced, secondary, radiated, reradiated, scattered, or outgoing. The scattering matrix, defined in the next section, will be seen to be a way of specifying the linear transformation from the first part of the field to the second.

The asymptotic relation between the far-field values of a scattered or radiated field and its spectrum is of essential interest and importance. This relation reveals the result of the interference among the waves of the continuous spectrum at large distances in any chosen direction of observation. Normally this interference results (remarkably) in the well-known exp $(i k r) / r$ variation with distance. This normal result does not apply to the spectrum of a plane wave, and it might not apply to the radiation or reradiation from a source of infinite size in one or more dimensions. For an antenna of finite size radiating into 3-dimensional space, we do have the asymptotic relations [33, p. 750$]$

$$
\begin{aligned}
& \mathbf{E}_{q t}^{r}(\mathbf{r}) \sim-i k|\cos \theta| \mathbf{B}_{q}(\mathbf{R} k / r) e^{i k r} / r \\
& \mathbf{E}_{q}^{r}(\mathbf{r}) \sim-i k|\cos \theta| \mathbf{b}_{q}(\mathbf{R} k / r) e^{i k r} / r
\end{aligned}
$$

Here the superscript " $r$ " refers to the radiated or reradiated component of the field and $\theta$ is the polar angle of $\mathbf{r}$ with respect to the $z$ axis. The first of the two equations relates to (14) and the second to (10); they differ only in the presence or absence of the $z$-component. Both equations are valid whether or not incident waves are also present in the field. Note that we have written $\mathbf{R} k / r$ for $\mathbf{K}$ as the argument of the spectral functions. This expresses the fact that the vectors $\mathbf{r}$ and $\mathbf{k}$ involved must be parallel. In fact if we introduce spherical coordinates for $\mathbf{r}$ such that $x=r \sin \theta$ $\cos \phi, y=r \sin \theta \sin \phi, z=r \cos \theta$, we see that $\mathbf{b}_{q}$ and $\mathbf{B}_{q}$ are expressed as functions of the angular coordinates of $\mathbf{r}$. Furthermore we note that here only real directions of propagation come into consideration, so that $\gamma$ is real and $\gamma=k|\cos \theta|$ is valid.

To conclude this subsection, we give a useful, nonphysical, special result, which offers a sharp contrast to the type of results given just above. Namely, if an electromagnetic field is every- 
where the (simple or evanescent) plane wave with $\mathbf{E}=\boldsymbol{\alpha} \exp \left(i \mathbf{k}_{\overline{0}} \cdot \mathbf{r}\right) / 2 \pi$, then from (9) the corresponding spectral functions are found to be

$$
\left.\begin{array}{l}
a_{1}(m, \mathbf{K})=b_{2}(m, \mathbf{K})=\alpha_{m} \delta\left(\mathbf{K}-\mathbf{K}_{0}\right), \\
b_{1}(m, \mathbf{K}) \equiv a_{2}(m, \mathbf{K}) \equiv 0,
\end{array}\right\}
$$

where $\alpha_{m} \equiv \boldsymbol{\kappa}_{m} \cdot \boldsymbol{\alpha}$ and $\delta\left(\mathbf{K}-\mathbf{K}_{0}\right)$ is an abbreviation for the delta-function product $\delta\left(k_{x}-k_{o x}\right)$ $\delta\left(k_{y}-k_{o y}\right)$. The vector

$$
\boldsymbol{A} \equiv \alpha_{1} \boldsymbol{\kappa}_{1}+\alpha_{2} \boldsymbol{\kappa}_{2}
$$

will be known as the transverse spectral vector associated with the considered plane wave.

\subsection{Definition of Scattering Matrices for Antennas and Scatterers}

Let us first consider that a passive material structure, exhibiting linear electromagnetic behavior, is present in the region $z_{1}<z<z_{2}$. The scattering equations are written

$$
b_{q}(m, \mathbf{K})=\sum_{p} \int_{\mathbf{L}} \sum_{n} S_{q p}(m, \mathbf{K} ; n, \mathbf{L}) a_{p}(n, \mathbf{L}) d \mathbf{L}, \quad(q=1,2)
$$

where, in addition to the summation and integration conventions noted following (1.2-8), we have summation over the index $p$, giving the contributions from the waves incident both from the "right" and from the "left." The processes described by the functions $S_{11}$ and $S_{22}$ will be called backscattering; those described by $S_{12}$ and $S_{21}$ will be called transcattering. Essentially the same definitions and an example of (1) may be found in [1].

If the scattering object is also an antenna, the scattering matrix must include the transmitting and receiving characteristics, and the scattering equations are written

$$
\begin{gathered}
b_{0}=S_{00} a_{0}+\sum_{p} \int_{\mathbf{L}} \sum_{n} S_{0 p}(n, \mathbf{L}) a_{p}(n, \mathbf{L}) d \mathbf{L}, \\
b_{q}(m, \mathbf{K})=S_{q 0}(m, \mathbf{K}) a_{0}+\sum_{p} \int_{\mathbf{L}} \sum_{n} S_{q p}(m, \mathbf{K} ; n, \mathbf{L}) a_{p}(n, \mathbf{L}) d \mathbf{L} .
\end{gathered}
$$

Here $q=1,2$ and we have made use of the quantities $b_{0}$ and $a_{0}$, defined in (1.1-1). The quantity $S_{00}$ represents "backscattering" observed at $S_{0}$ in the feed waveguide, and the functions $S_{0 q}(m, \mathbf{K})$ and $S_{q 0}(m, \mathbf{K})$ respectively represent the receiving and the transmitting characteristics of the antenna. (The quantities bearing the subscripts $q$ or $p=2$ in (2) represent the desired generalization of the antenna scattering matrix originally defined by Kerns and Dayhoff [1].)

The definition of the antenna scattering matrix is now literally complete. We do not wish to belabor the generality of the definition, but we do call attention to the absence of restrictive assumptions. At best, its full significance can be made apparent only gradually.

It will be advantageous to have the basic scattering equations expressed in vector-dyadic form. In addition to the transverse vectors $\mathbf{B}_{q}$ and $\mathbf{A}_{q}$ defined in (1.2-15), we introduce the vectorial transmitting characteristic

$$
\mathbf{S}_{q 0}(\mathbf{K})=\sum_{m} S_{q 0}(m, \mathbf{K}) \boldsymbol{\kappa}_{m},
$$


the vectorial receiving characteristic

$$
\mathbf{S}_{0 q}(\mathbf{K})=\sum_{m} S_{0 q}(m, \mathbf{K}) \boldsymbol{\kappa}_{m},
$$

and the dyadic scattering characteristic

$$
\mathbf{S}_{q p}(\mathbf{K}, \mathbf{L})=\sum_{m, n} S_{q p}(m, \mathbf{K} ; n, \mathbf{L}) \boldsymbol{\kappa}_{m} \boldsymbol{\lambda}_{n},
$$

where the $\boldsymbol{\kappa}_{m}$ and $\boldsymbol{\lambda}_{n}$ are the unit vectors associated respectively with $\mathbf{K}$ and $\mathbf{L}$. The scattering equations become

$$
\begin{gathered}
b_{0}=S_{00} a_{0}+\sum_{p} \int \mathbf{S}_{0 p}(\mathbf{K}) \cdot \mathbf{A}_{p}(\mathbf{K}) d \mathbf{K} \\
\mathbf{B}_{q}(\mathbf{K})=\mathbf{S}_{q 0}(\mathbf{K}) a_{0}+\sum_{p} \int \mathbf{S}_{q p}(\mathbf{K}, \mathbf{L}) \cdot \mathbf{A}_{p}(\mathbf{L}) d \mathbf{L} .
\end{gathered}
$$

The equations are now invariant with respect to choice of coordinates in the transverse plane. This facilitates discussion of "real world" cases, in which it is neither expedient nor necessary always to observe or produce pure $\boldsymbol{\kappa}_{1}$ or $\boldsymbol{\kappa}_{2}$, field components.

One may obtain an analysis of the scattering equations by considering the simplest modes of excitation: by a wave represented by $a_{0}$ alone, and by individual spatial plane waves, represented by delta-function spectra. This procedure leads essentially to re-expressions of the basic definitions contained in the scattering equations. The circumstances of the resulting definitions or expressions are simple enough to suggest several more or less direct and conventional methods of measurement for the scattering-matrix elements. Let us in particular consider the definitions of $\mathbf{S}_{q 0}(\mathbf{K})$ and $\mathbf{S}_{0 q}(\mathbf{K})$ - the scattering-matrix quantities that will receive the most attention in our work.

Let us consider an antenna operating in its transmitting mode; that is, an antenna excited only by an incident wave in its waveguide feed and radiating into empty space. This elementary pattern of excitation is represented by $a_{0} \neq 0, a_{q}(m, \mathbf{K}) \equiv 0$. Under these conditions, the spectra radiated (to the left and to the right), normalized to unit $a_{0}$, characterize the transmitting properties of the antenna; indeed, from $(4 \mathrm{~b})$

$$
\mathbf{S}_{q 0}(\mathbf{K})=\frac{\mathbf{B}_{q}(\mathbf{K})}{a_{0}} .
$$

Incident spatial waves being absent, $(1.2-9 \mathrm{a})$ and $(1.2-9 \mathrm{~b})$ together imply that $\mathbf{B}_{q}$ may be related either to $\mathbf{E}$ or to $\mathbf{H}$; in terms of $\mathbf{E}$ we have

$$
\mathbf{S}_{q 0}(\mathbf{K})=\frac{e^{-i \gamma|z|}}{2 \pi a_{0}} \int \mathbf{E}_{q t}(\mathbf{R}, z) e^{-i \mathbf{K} \cdot \mathbf{R}} d \mathbf{R}
$$

where $z>z_{1}$ or $z<z_{2}$ must be in force. This gives us a Fourier transform definition of $\mathbf{S}_{q 0}$, and, to the extent that $\mathbf{E}_{q t} / a_{0}$ is known or measurable, a means of calculating $\mathbf{S}_{q 0}$. The asymptotic relation (1.2-16a), applied in the present circumstances, may be written

$$
\mathbf{E}_{q t}(\mathbf{r}) \sim-i \gamma \mathbf{S}_{q 0}(\mathbf{R} k / r) a_{0} e^{i k r} / r .
$$

Clearly this relation may be regarded as a formula for determining $\mathbf{S}_{q 0}(\mathbf{K})$ in terms of observed asymptotic $\mathbf{E}_{q t} / a_{0}$. It in fact represents the basis of so-called direct methods of measurement of transmitting characteristics. 
The combination of (6) and (7) incidentally furnishes a rigorous, vectorial form of the Fouriertransform relation between far- and near-fields.

Next, consider an antenna excited solely by a spatial plane wave, incident on side $q$, say, and having $\mathbf{E}=\boldsymbol{\alpha} \exp (i \mathbf{k} \cdot \mathbf{r}) / 2 \pi$. The pattern of excitation is accordingly represented by $a_{0}=0$ and $a_{q^{\prime}}\left(m, \mathbf{K}^{\prime}\right)=\alpha_{m} \delta_{q^{\prime} q} \delta\left(\mathbf{K}^{\prime}-\mathbf{K}\right)$, where $\alpha_{m} \equiv \boldsymbol{\kappa}_{m} \cdot \boldsymbol{\alpha}$ and the spectrum of the incident wave is found just as in (1.2-17). The scattering equations (2a) or (4a) now yield

$$
\begin{aligned}
b_{0} & =S_{0 q}(1, \mathbf{K}) \alpha_{1}+S_{0 q}(2, \mathbf{K}) \alpha_{2}, \\
& =\mathbf{S}_{0 q}(\mathbf{K}) \cdot \boldsymbol{A} .
\end{aligned}
$$

In other words, $S_{0 q}(m, \mathbf{K})$ denotes the receiving sensitivity-or receptivity-of the antenna to the $\boldsymbol{\kappa}_{m}$-component of polarization of a plane wave incident on the antenna with direction of incidence specified by $q$ and $\mathbf{K}$. The normalization is to unit $\alpha_{m}$ (which means unit delta-function spectrum amplitude).

Equation (8) immediately suggests basic equations for direct measurement of receiving characteristics. Viz.,

$$
\begin{aligned}
& b_{0}^{\prime}=\mathbf{S}_{0 q}(\mathbf{K}) \cdot \boldsymbol{A}^{\prime}, \\
& b^{\prime \prime}=\mathbf{S}_{0 q}(\mathbf{K}) \cdot \boldsymbol{A}^{\prime \prime},
\end{aligned}
$$

where $b_{0}^{\prime}, b_{0}^{\prime \prime}$ are observed and $\boldsymbol{A}^{\prime}, \boldsymbol{A}^{\prime \prime}$ are known and linearly independent. The equation can, of course, be solved for $\mathbf{S}_{0 q}$; an algebraically identical problem is encountered in subsection 4.1.

The following compact notation for the scattering equations, to be secured by introducing function vectors and making more use of matrix-algebraic concepts, provides a perspective quite different from that of the discussion just preceding. We first define the column matrices

$$
\left(\begin{array}{c}
a_{0} \\
\hat{a}_{1} \\
\hat{a}_{2}
\end{array}\right), \quad\left(\begin{array}{l}
b_{0} \\
\hat{b}_{1} \\
\hat{b}_{2}
\end{array}\right),
$$

in which $\hat{a}_{q}$ and $\hat{b}_{q}$ may themselves be regarded as column matrices representing the functions $a_{q}(m, \mathbf{K})$ and $b_{q}(m, \mathbf{K})$. That is to say, the elements of these column matrices are labeled or indexed according to the values of $m$ and $\mathbf{K}$, and have the values $a_{q}(m, \mathbf{K})$ and $b_{q}(m, \mathbf{K})$, respectively. The transformation from the entire set of incident waves to the entire set of emergent waves is now written

$$
\left(\begin{array}{c}
b_{0} \\
\hat{b}_{1} \\
\hat{b}_{2}
\end{array}\right)=\left(\begin{array}{lll}
S_{00} & \hat{S}_{01} & \hat{S}_{02} \\
\hat{S}_{10} & \hat{S}_{11} & \hat{S}_{12} \\
\hat{S}_{20} & \hat{S}_{21} & \hat{S}_{22}
\end{array}\right) \quad\left(\begin{array}{c}
a_{0} \\
\hat{a}_{1} \\
\hat{a}_{2}
\end{array}\right)
$$

or, equivalently, after performing the indicated matrix multiplication,

$$
\begin{aligned}
& b_{0}=S_{00} a_{0}+\hat{S}_{01} \hat{a}_{1}+\hat{S}_{02} \hat{a}_{2}, \\
& \hat{b}_{1}=\hat{S}_{10} a_{0}+\hat{S}_{11} \hat{a}_{1}+\hat{S}_{12} \hat{a}_{2}, \\
& \hat{b}_{2}=\hat{S}_{20} a_{0}+\hat{S}_{21} \hat{a}_{1}+\hat{S}_{22} \hat{a}_{2} .
\end{aligned}
$$


Here the four kinds of products involved are defined by comparison with (2) or (4). This compact notation, in which the subscripts identify the three input-output reference surfaces, makes the overall structure of the equations more apparent and is a practical necessity for the demonstration in the next section. The rules of matrix algebra apply: the $\hat{S}_{q 0}$ (as well as $\hat{a}_{q}$ and $\hat{b}_{q}$ ) correspond to column matrices; the $\hat{S}_{0 q}$, to row matrices; and the $\hat{S}_{p q}$, to square matrices.

We should notice the form that the scattering equations take in the absence of any scattering object (or conceivably in the presence of a non-scattering object). The free passage of waves is expressed by

$$
b_{1}(m, \mathbf{K}) \equiv a_{2}(m, \mathbf{K}), \quad b_{2}(m, \mathbf{K}) \equiv a_{1}(m, \mathbf{K}) .
$$

(No propagation factors of the form $\exp ( \pm i \gamma d)$ appear here because the phase reference surfaces were chosen coincident at $z=0$ ). The pertinent submatrix in (11) is

$$
\left(\begin{array}{ll}
\hat{S}_{11} & \hat{S}_{12} \\
\hat{S}_{21} & \hat{S}_{22}
\end{array}\right)=\left(\begin{array}{ll}
\hat{0} & \hat{1} \\
\hat{1} & \hat{0}
\end{array}\right)
$$

Here $\hat{1}$ denotes the identity transformation with elements $\delta_{m n} \delta\left(k_{x}-l_{x}\right) \delta\left(k_{y}-l_{y}\right)$ and $\hat{0}$ denotes the zero transformation. One may regard a scattering object as producing a perturbation of the properties of free-space described in (13) or (14). From this viewpoint, what we may call true or bona fide transcattering is described by the operators $\hat{S}_{12}-\hat{1}$ and $\hat{S}_{21}-\hat{1}$.

\subsection{Power Expressions}

The requisite expression for one-mode power transfer in the antenna feed waveguide was given in (1.1-4). Here we shall give the corresponding expressions for power transfer across the surfaces $F_{1}$ and $F_{2}$.

The time-average energy flux across the surface $F_{q}$ in the outward direction (the direction of $\mathbf{n}_{q}$ ) is given by

$$
P_{q}=\frac{1}{2} \operatorname{Re} \int_{F q}\left[\mathbf{E} \overline{\mathbf{H}} \mathbf{n}_{q}\right] d \mathbf{R},
$$

where the integrand is the scalar triple product and the superposed bar denotes the complex conjugate. We wish to evaluate $P_{q}$ in terms of the spectral density functions $a_{q}(m, \mathbf{K})$ and $b_{q}(m, \mathbf{K})$. After some analysis, one obtains

$$
P_{q}=\frac{1}{2} \int_{k<k} \sum_{m}\left(\left|b_{q}\right|^{2}-\left|a_{q}\right|^{2}\right) \eta_{m} d \mathbf{K}-\int_{k>k} \sum_{m} \operatorname{Re}\left(\bar{b}_{q} a_{q} \eta_{m}\right) d \mathbf{K},
$$

noting that for the lossless medium $\gamma$ and $\eta_{m}$ are both real in the propagating region $(K<k)$ and both imaginary in the evanescent region $(K>k)$. The equation shows that power may be transferred by the coupling of incoming and outgoing evanescent modes having the same $m$ and K. (This the essential mechanism of power transfer in a waveguide-below-cutoff attenuator.) We expect and assume that ordinarily this interaction will be negligible, as in the case of coupling of ordinary waveguide junctions; the separation required is measured in wavelengths, not Rayleigh distances. Thus the basic expression (2) may ordinarily be abbreviated to

$$
P_{q}=\frac{1}{2} \int_{K<k} \sum_{m}\left[\left|b_{q}(m, \mathbf{K})\right|^{2}-\left|a_{q}(m, \mathbf{K})\right|^{2}\right] \eta_{m}(K) d \mathbf{K} .
$$


This may be used, e.g., to deduce conservation relations. In the absence of incident waves, (3) expresses the equivalent of "pattern integration" over a hemisphere.

\subsection{Reciprocal Relations-A Summary}

In this section we briefly state recently derived [34] generalized or adjoint reciprocity relations for antennas and scatterers. (The derivation is reproduced in appendix A.) These relations very readily adapt to the expression of ordinary reciprocity as a special case. We shall also comment on the question of "realizability" of mutually adjoint systems. First we must define mutually adjoint media and systems.

We describe the distribution of material media making up an antenna or scattering structure by means of the constitutive equations

$$
\mathbf{D}=\boldsymbol{\epsilon} \cdot \mathbf{E}+\boldsymbol{\tau} \cdot \mathbf{H}, \quad \mathbf{B}=\boldsymbol{\nu} \cdot \mathbf{E}+\boldsymbol{\mu} \cdot \mathbf{H}
$$

Here the tensors $\boldsymbol{\epsilon}$ and $\boldsymbol{\mu}$ have their usual roles; $\boldsymbol{\tau}$ and $\boldsymbol{\nu}$ allow for the description of possible magnetoelectric properties of the medium [35]. ${ }^{5}$ (This last bit of generality may provide future benefits and does not appreciably complicate the discussion.) The tensor parameters will of course in general depend upon position within the region of the antenna or scatterer considered; outside this region the set of parameters must reduce nominally to vacuum values: $\epsilon=\epsilon_{0}, \boldsymbol{\mu}=\mu_{0}, \boldsymbol{\tau}=\boldsymbol{\nu}=0$.

In addition to a "given" or "original" system, described by the above equations, we must consider the adjoint system, which is described by the constitutive equations for the adjoint medium, ${ }^{6}$

$$
\mathbf{D}=\tilde{\boldsymbol{\epsilon}} \cdot \mathbf{E}-\tilde{\boldsymbol{\nu}} \cdot \mathbf{H}, \quad \mathbf{B}=-\tilde{\boldsymbol{\tau}} \cdot \mathbf{E}+\tilde{\boldsymbol{\mu}} \cdot \mathbf{H},
$$

where the superposed tilde denotes the transpose, and the tensors $\boldsymbol{\epsilon}, \boldsymbol{\tau}, \boldsymbol{\mu}$, and $\boldsymbol{\nu}$ are those of the original system. As the equations show, "adjointness" is a mutual relationship: the adjoint of the adjoint system is the original system.

Since the tensor parameters are essentially arbitrary, we should realize that the "original" system is in no way a theoretically preferred system; the designation is arbitrary but useful.

The concept that a medium may be lossy, lossless, or even "gainy" is familiar. In an inhomogeneous medium these "dissipative properties" will in general change from point to point. In appendix B dissipative properties are more precisely defined and it is shown that these properties are point-wise identical for mutually adjoint media. In view of the usual connection between dissipative properties and the concept of "realizability," we may say that mutually adjoint media are equally realizable.

Nonreciprocal antennas are most commonly (if not invariably) so because of the use of ferrites subjected to a static magnetic biasing field. In such cases the adjoint antenna can in principle be produced by reversal of the bias field. (We say "in principle" because in general no provision is made for conveniently or precisely accomplishing the bias field reversal.)

The scattering matrix for the adjoint antenna may be and is assumed to be defined with the same basis fields, the same reference surfaces, and altogether in the same way as that for the original antenna. Then, as shown in appendix A, the following generalized or adjoint reciprocity relations hold between the characteristics of mutually adjoint antennas: For the antenna-feed reflection coefficients,

$$
S_{00}=S_{00}^{a}
$$

${ }^{5}$ In some of the recent literature, media described by equations of the form of (1) are called "bianisotropic." However, optically active media, which have been studied for many years [36], satisfy equations of the above form with scalar parameters and are not anisotropic at all. Optically active media incidentally are reciprocal, and thus could form part of a reciprocal antenna or other device.

${ }^{6}$ Media related by (1) and (2) are called "complementary" media by Kong and Cheng [37]. The adjective "adjoint" seems more appropriate both nontechnically and technically: Maxwell's equations for the adjoint system can be written as the mathematical adjoint of Maxwell's equations for the original system. See appendix A. 
for the transmitting and the receiving characteristics,

$$
\begin{aligned}
& \eta_{0} S_{0 q}^{a}(m, \mathbf{K})=-\eta_{m}(K) S_{q 0}(m,-\mathbf{K}), \quad(q=1,2) \\
& \eta_{0} S_{0 q}(m, \mathbf{K})=-\eta_{m}(K) S_{q 0}^{a}(m,-\mathbf{K}) ; \quad(q=1,2)
\end{aligned}
$$

and for the scattering characteristics,

$$
\eta_{m}(K) S_{p q}(m, \mathbf{K} ; n, \mathbf{L})=\eta_{n}(L) S_{p q}^{a}(n,-\mathbf{L} ; m,-\mathbf{K}) . \quad(p=1,2 ; q=1,2)
$$

The superscript " $a$ " distinguishes quantities associated with the adjoint antenna. We observe that all the equations hold with $S$ and $S^{a}$ interchanged.

Scattering-matrix elements, such as $S_{10}(m, \mathbf{K})$ and $S_{11}(m, \mathbf{K}, n, \mathbf{L})$, characterize processes associated with a pair of directions: ${ }^{7}$ the direction of an incident wave and a direction of "observation." For the functions mentioned, the direction-pairs are $\mathbf{n}_{0}, \mathbf{e}_{k}$ and $\mathbf{e}_{l}, \mathbf{e}_{k}$, respectively. Reciprocity relates processes associated with two pairs of directions, obtained by reversing and interchanging the direction of incidence and the direction of observation. See figures 4 and 5 .

If the constitutive tensors obey the symmetry relations

$$
\boldsymbol{\epsilon}=\tilde{\boldsymbol{\epsilon}}, \boldsymbol{\mu}=\tilde{\boldsymbol{\mu}} \text {, and } \boldsymbol{\tau}=-\tilde{\boldsymbol{\nu}}
$$

then, as may be seen directly from (1) and (2), the adjoint antenna and the original antenna are identical. In this case, if we use conventional terminology, we say that the original antenna is reciprocal; in the present context, a term such as self-reciprocal or self-adjoint would be less liable to ambiguity. In the self-adjoint case the superscript " $a$ " is without force and may be eliminated; (3), (4), and (5) become expressions of properties of one and the same antenna (and reduce to results given with $p=q=1$ in [1]).

Equations (3), (4), and (5) are our basic expressions of reciprocity and adjoint reciprocity. As mentioned in the Introduction, these relations have found substantial application in research establishing the foundations of the extrapolation technique [6,7]. More immediate consequences are brought out in the next subsection and in section 4 .
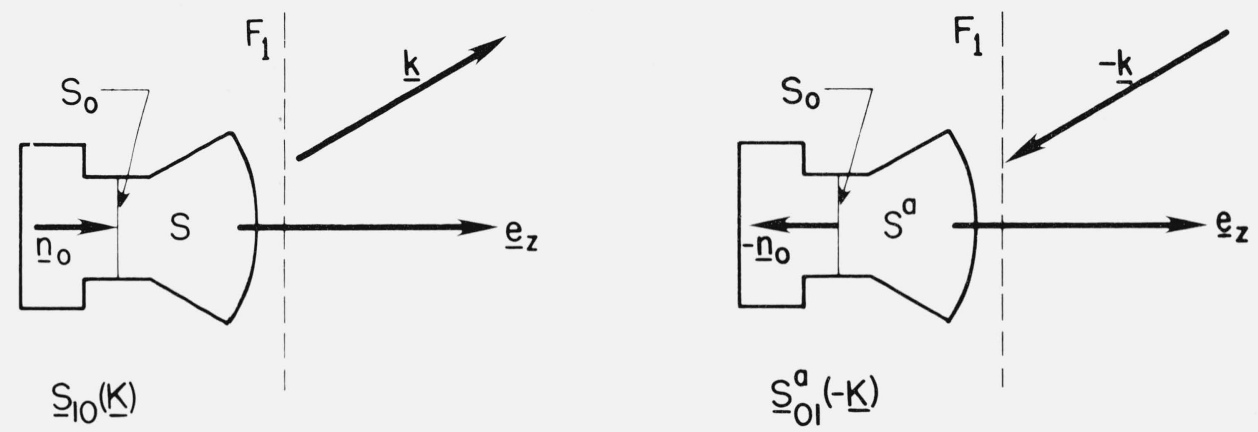

FIGURE 4. Reciprocity direction-diagram for $\mathbf{S}_{10}$ and $\mathbf{S}_{01}^{a}$.

${ }^{7}$ This is in marked contrast to what is involved when spherical or other nonplanar waves are used to represent the fields in space. 

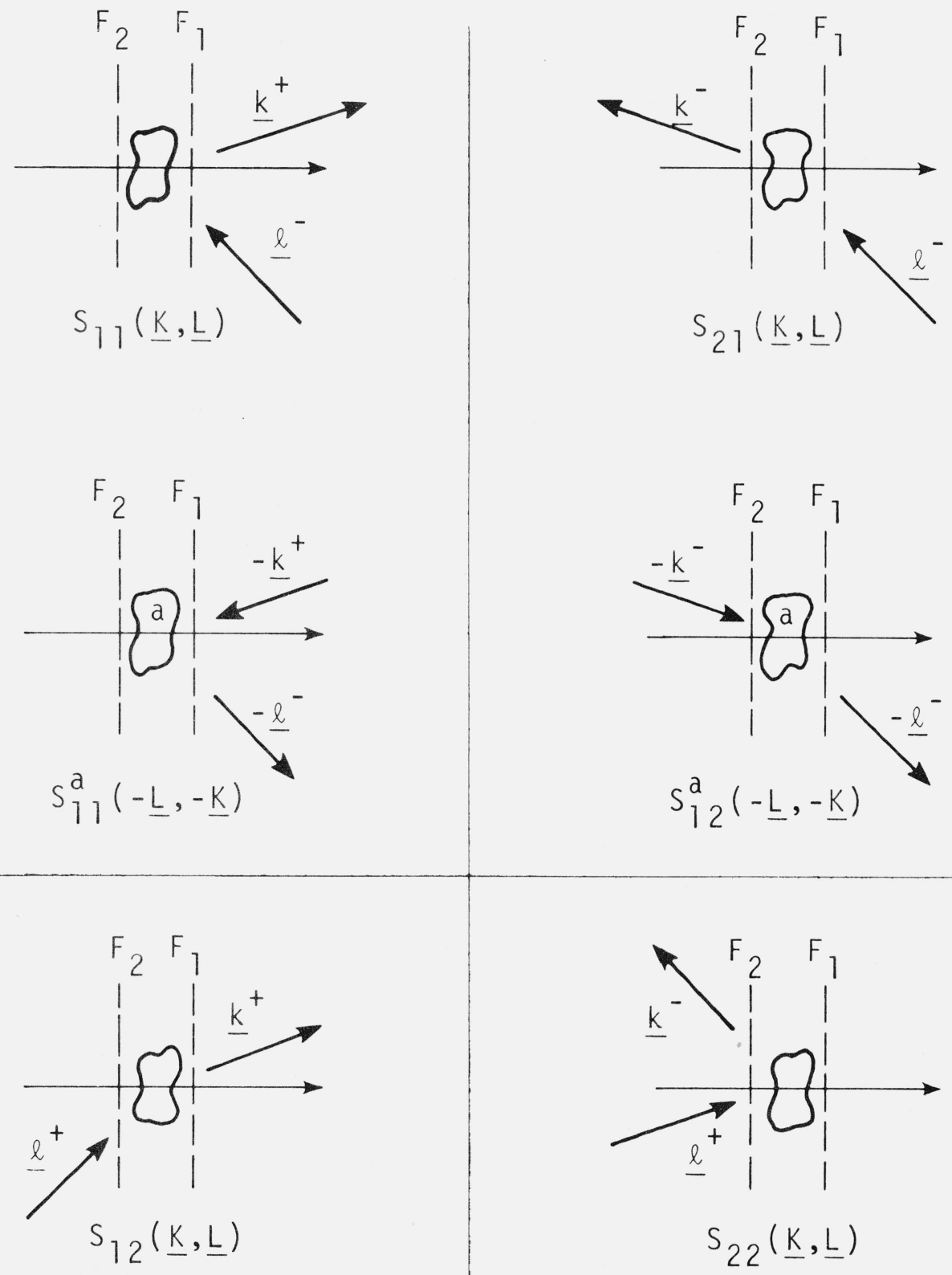

$$
S_{22}(\underline{K}, \underline{L})
$$
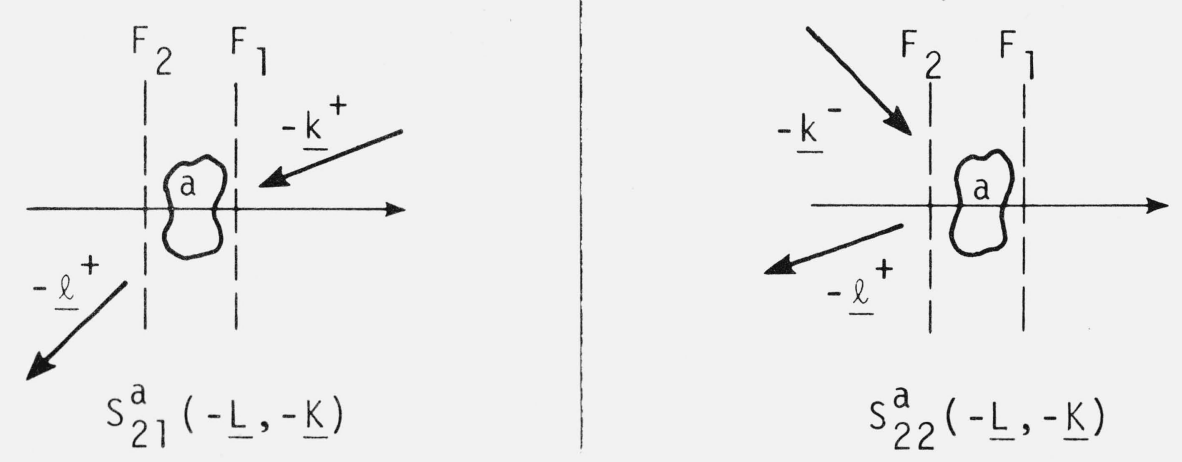

FigURE 5. Direction-diagrams for the scattering reciprocity relations. 


\subsection{Power Gain, Effective Area, and Polarization Indices}

Our primary purpose in this subsection is to exhibit some of the content and applicability of the PWSM formalism by defining some of the more familiar and conventional quantities of antenna theory in terms of the antenna scattering matrix quantities. In particular, we shall define the powergain function $G_{q}$ and the effective-area function $\sigma_{q}$ associated respectively with the transmitting and receiving characteristics $\mathbf{S}_{q 0}$ and $\mathbf{S}_{0 q}$. We shall also define polarization indices $\rho_{q 0}$ and $\rho_{0 q}$ associated respectively with $\mathbf{S}_{q 0}$ and $\mathbf{S}_{0 q}$. All the quantities to be defined are of course functions of direction and all are meaningful and applicable in dealing with the coupling of widely separated antennas, the context of conventional antenna theory. It will be seen, however, that although these quantities are uniquely defined (in a given coordinate system) by the antenna scattering-matrix quantities, the converse is not true. Hence they are not adequate characterizations of transmitting and receiving properties for the type of theory of essential interest in this paper (and, of course, they say nothing about scattering properties of an antenna).

Our definitions of power gain and effective area are consistent with the essential content of the corresponding IEEE Standard definitions. However, our definitions of receiving and of transmitting properties are formed wholly independently of each other-in contrast to a tactic used to some extent in the IEEE Standards. Reciprocity relations then appear only later in their proper role as theorems.

The key relation appropriate for the present consideration of transmitting characteristics is the asymptotic relation

$$
\mathbf{E}_{q}(\mathbf{r}) \sim-i \gamma \mathbf{s}_{q^{0}}(\mathbf{R} / i / r) a_{0} e^{i k r / r} .
$$

Here

$$
\mathbf{s}_{q 0}(\mathbf{K}) a_{0}=\mathbf{b}_{q}(\mathbf{K}),
$$

where $\mathbf{b}_{q}$ is the complete radiated spectrum in the sense of $(1.2-12 \mathbf{a})$ and $\mathbf{s}_{q 0}$ is the corresponding "complete" transmitting characteristic given in terms of components of $\mathbf{S}_{0 q}$ by

$$
\mathbf{s}_{q 0}(\mathbf{K})=\left[(-)^{q-1} l_{i} / \gamma\right] S_{q 0}(1, \mathbf{K}) \mathbf{e}_{\|}+S_{q 0}(2, \mathbf{K}) \mathbf{e}_{\perp} .
$$

The following relations are noted:

$$
\mathbf{k}^{ \pm} \cdot \mathbf{s}_{q 0}(\mathbf{K})=0, \quad \mathbf{s}_{q 0}(0)=\mathbf{S}_{q 0}(0),
$$

and $\mathbf{S}_{q 0}(\mathbf{K})$ is the projection of $\mathbf{s}_{q 0}(\mathbf{K})$ on the $k_{x}, k_{y}$ plane.

From (1) one easily finds for the power radiated per unit solid angle at large distances

$$
p_{q}=\frac{1}{2} Y_{0} \gamma^{2}\left|\mathbf{s}_{q_{0}}(\mathbf{K}) a_{0}\right|^{2} .
$$

The $Y_{0}$ appearing here is the value of the admittance $\left(\epsilon_{0} / \mu_{0}\right)^{1 / 2}$ for the ambient medium. The function $p_{q}$ is a "power pattern" for the considered antenna; generally any function proportional to $p_{q}$, whether or not the factor of proportionality is known, is called a power pattern.

The power gain (function) of an antenna is defined by

$$
G_{q}(\mathbf{K})=4 \pi p_{q} / P_{0},
$$


where $P_{0}$ is the net input power to the antenna. It follows from (1.1-4) and (5) that

$$
G_{q}(\mathbf{K})=\frac{4 \pi Y_{0} \gamma^{2}\left|\mathbf{s}_{q 0}(\mathbf{K})\right|^{2}}{\eta_{0}\left(1-\left|S_{00}\right|^{2}\right)} .
$$

It should be noted that the power gain is a characteristic of the antenna under consideration, independent of the source used to excite the antenna. This means that the value of $G_{q}(\mathbf{K})$ is independent of the insertion or adjustment of a lossless tuner in the feed waveguide, whether or not this tuner is counted as part of the antenna (cf. discussion of eq (2.6-13) in [30]).

Using the components shown in (3), we define the polarization index

$$
\rho_{q 0}(\mathbf{K})=\frac{S_{q 0}(2, \mathbf{K})}{S_{q 0}(1, \mathbf{K})(-)^{q-1} k / \gamma}
$$

associated with the transmitting characteristics of an antenna. This spectral polarization index is definable more physically and more conventionally in terms of the components of the corresponding asymptotic $\mathbf{E}$; by (1), it is just the ratio of the $\perp$ and $\|$ (or $\phi$ and $\theta$ ) components of this $\mathbf{E}$ in the direction of observation. Polarization characteristics are conveniently and fully described by the single complex number $\rho_{q 0}$; other polarization parameters, such as axial ratio and orientation of the associated polarization ellipse, can be determined, if desired, from $\rho_{q 0}$.

For an antenna functioning in a receiving mode, the counterpart of the power gain is the effective area or effective receiving cross-section, $\sigma_{q}(\mathbf{K})$. Like the gain, this quantity is a scalar function of direction and involves a far-field concept - in this case that of an incident plane wave. It is here defined by

$$
P_{A, \max }=\sigma_{q}(\mathbf{K}) S_{P o y},
$$

where $P_{A, \max }$ is the available power at the antenna terminal and $S_{P o y}$ is the magnitude of the Poynting's vector of a plane wave arriving at the antenna from a given direction and providing a polarization match to the antenna receiving characteristic. The derivation of an expression for $\sigma_{q}$ involves a number of intermediate results that are at least as important as $\sigma_{q}$ itself.

Let the electric field of the wave incident on side $q$ of the antenna be $\mathbf{E}=\boldsymbol{\alpha} \exp (i \mathbf{k} \mathbf{r}) / 2 \pi$; then from $(1.3-2 \mathrm{a})$ or $(1.3-4 \mathrm{a})$, the emergent wave-amplitude at the antenna terminal is

$$
b_{0}=S_{00} a_{0}+\mathbf{S}_{0 q}(\mathbf{K}) \cdot \boldsymbol{A}
$$

(This expression differs from (1.3-8) only in that here we have not assumed $a_{0}=0$.) Thus, from (9), we see that the antenna, as excited by the incident spatial wave, appears at $S_{0}$ as a source having a reflection coefficient $S_{00}$ and a generated wave $b_{G}=S_{0 q}(\mathbf{K}) \cdot \boldsymbol{A}$. By ordinary (microwave) circuit calculation the corresponding received power is found to be

$$
P_{\mathrm{rec}}=\frac{1}{2} \eta_{0} \frac{\left(1-\left|\Gamma_{L}\right|^{2}\right)\left|\mathbf{S}_{0 q}(\mathbf{K}) \cdot \boldsymbol{A}\right|^{2}}{\left|1-\Gamma_{L} S_{00}\right|^{2}}
$$

where $\Gamma_{L}$ is the reflection coefficient of the passive termination at $S_{0}$. By setting $\Gamma_{L}=\bar{S}_{00}$, we get for the available power

$$
P_{A}=\frac{1}{2} \eta_{0} \frac{\left|\mathbf{S}_{0 q}(\mathbf{K}) \cdot \boldsymbol{A}\right|^{2}}{1-\left|S_{00}\right|^{2}}
$$

To aid in the consideration of the polarization-related parts of the problem, we display the spectral vector

$$
\boldsymbol{A}=\alpha_{1} \boldsymbol{\kappa}_{1}+\alpha_{2} \boldsymbol{\kappa}_{2}
$$


used here and originally defined in (1.2-18); we introduce the complete spectral vector for the incident wave in the form

$$
\boldsymbol{\alpha}=\left[(-)^{q} k / \gamma\right] \alpha_{1} \mathbf{e}_{\|}+\alpha_{2} \mathbf{e}_{\perp}
$$

and we define the polarization index

for the incident wave.

$$
w_{q}(\mathbf{K}) \equiv \frac{\alpha_{2}}{\alpha_{1}(-)^{q} k / \gamma}
$$

Further, it is convenient to define a receiving characteristic, $\mathbf{s}_{0 q}$, complementary to the complete transmitting characteristic $\mathbf{s}_{q 0}$. The single essential requirement is that

$$
\mathbf{s}_{0 q}(\mathbf{K}) \cdot \boldsymbol{\alpha}=\mathbf{S}_{0 q}(\mathbf{K}) \cdot \boldsymbol{A}
$$

be an identity in $\boldsymbol{A}$. This leaves a possible $\mathbf{e}_{k}$-component of $\mathbf{s}_{0 q}$ undefined; we are in fact free to require

$$
\mathbf{k}^{\mp} \cdot \mathbf{s}_{0 q}(\mathbf{K}) \equiv 0 .
$$

(Here, as before, the upper sign goes with $q=1$, the lower, with $q=2$.) Equations (14) and (12) imply

$$
\mathbf{s}_{0 q}(\mathbf{K})=\left[(-)^{q} \gamma / k\right] S_{0 q}(1, \mathbf{K}) \mathbf{e}_{\|}+S_{0 q}(2, \mathbf{K}) \mathbf{e}_{\perp} .
$$

One should note particularly that $\gamma / k$ appears here whereas $k / \gamma$ appears in the expression for $\mathbf{s}_{q_{0}}$; $\mathbf{s}_{0 q}$ is in no way a "complete" vector of which $\mathbf{S}_{0 q}$ is a part. However, the relation

does hold.

$$
\mathbf{s}_{0 q}(0)=\mathbf{S}_{0 q}(0)
$$

Using the components shown in (15), we define a polarization index for the receiving characteristics

$$
\rho_{0 q}(\mathbf{K}) \equiv \frac{S_{0 q}(2, \mathbf{K})}{S_{0 q}(1, \mathbf{K})(-)^{q} \gamma / k} .
$$

This parameter relates to the properties of a passive material structure; it does not directly characterize the elliptical path of a time-varying vector. It is a ratio of receptivities to components of polarization in an incident plane wave under the specified conditions.

Observing that $|\boldsymbol{\alpha}|^{2}=8 \pi^{2} S_{\text {Poy }} / Y_{0}$, we can now combine (11), (14a), (13), and (16) to obtain

$$
P_{A}=\left[\frac{\left|1+w_{q} \rho_{0 q}\right|^{2}}{\left(1+\left|w_{q}\right|^{2}\right)\left(1+\left|\rho_{0 q}\right|^{2}\right)}\right] \frac{4 \pi^{2} \eta_{0}\left|\mathbf{s}_{o q}\right|^{2} S_{P 0 y}}{Y_{0}\left(1-\left|S_{00}\right|^{2}\right)}
$$

The quantity in brackets is a "polarization mismatch" factor, which, according to the Schwarz inequality for complex vectors, attains its maximum value of unity for

$$
w_{q}(\mathbf{K})=\overline{\rho_{0 q}(\mathbf{K})}
$$

Thus the condition for polarization match is expressed as a conjugate match of polarization indices. When (18) holds, we find from (17) and (8)

$$
\sigma_{q}(\mathbf{K})=\frac{4 \pi^{2} \eta_{0}\left|\mathbf{s}_{0 q}(\mathbf{K})\right|^{2}}{Y_{0}\left(1-\left|S_{00}\right|^{2}\right)}
$$

as the desired expression for the effective area. 
The minimum value of $P_{A}$, incidentally, is zero: for the given antenna and for any given direction of the incident wave, there is always a wave-polarization, $w_{q}=-1 / \rho_{0 q}$, that is not received at all. The polarizations best received and not received are mutually orthogonal (in the power or Hermitian sense).

The complete transmitting characteristic $\mathbf{s}_{q 0}$ and the complementary receiving characteristic $\mathbf{s}_{0 q}$ for a reciprocal antenna satisfy the reciprocity relation

$$
\eta_{0} k \mathbf{s}_{0 q}(\mathbf{K})=Y_{0} \gamma \mathbf{s}_{q 0}(-\mathbf{K})
$$

which follows from the basic relations (1.5-4). (In applying (1.5-4) it may be convenient to use the forms $\eta_{1}=Y_{0} t_{i} / \gamma$ and $\eta_{2}=Y_{0} \gamma / l_{i}$ for the $\eta$ 's, which were originally defined in $(1.2-5,-6)$.) For mutually adjoint antennas, the relations corresponding to (20a) are

$$
\eta_{0} h_{\mathbf{s}_{0 q}^{a}}^{a}(\mathbf{K})=Y_{0} \gamma \mathbf{s}_{q 0}(-\mathbf{K}), \quad \eta_{0} h_{\mathbf{s}_{0 q}}(\mathbf{K})=Y_{0} \gamma \mathbf{s}_{q 0}^{a}(-\mathbf{K})
$$

The power-gain and effective-area functions for a reciprocal antenna satisfy the well-known reciprocity relation

$$
\sigma_{q}(\mathbf{K})=\frac{\lambda^{2}}{4 \pi} G_{q}(-\mathbf{K})
$$

For mutually adjoint antennas the corresponding relations are

$$
\sigma_{q}^{a}(\mathbf{K})=\frac{\lambda^{2}}{4 \pi} G_{q}(-\mathbf{K}), \quad \sigma_{q}(\mathbf{K})=\frac{\lambda^{2}}{4 \pi} G_{q}^{a}(-\mathbf{K}) .
$$

The results in this set are conveniently found as corollaries of (20). Relations of the type (21b) were noted by Harrington and Villeneuve for antennas containing gyrotropic media [38].

The reciprocity constraint for the transmitting and receiving polarization characteristics of a reciprocal antenna reads

$$
\rho_{0 q}(-\mathbf{K})=-\rho_{q 0}(\mathbf{K})
$$

The corresponding relations for mutually adjoint antennas are

$$
\rho_{0 q}^{a}(-\mathbf{K})=-\rho_{q 0}(\mathbf{K}), \quad \rho_{0 q}(-\mathbf{K})=-\rho_{q 0}^{a}(\mathbf{K}),
$$

which follow as further consequences of (1.5-4) or (20).

An interesting corollary of the polarization matching and reciprocity theorems is that if the radiation from a reciprocal antenna in a certain direction is circularly polarized, the wave best received from that direction is circularly polarized in the same screw sense. A verification of this, though basically simple, involves a few key elements. The polarization-index definitions (7) and (13) are so fashioned that $\rho_{q 0}(\mathbf{K})=i$ and $w_{q}(\mathbf{K})=i$ both represent right-handed circular polarizations ${ }^{8}$ (for $q=1,2$ and for all $K<h_{i}$ ). Here the behavior, and in particular the parity, of the unit vectors $\mathbf{e}_{\|}$and $\mathbf{e}_{1}$ as functions of $\mathbf{k}$ is directly involved (refer to table 1 and especially to fig. 6). Note that $\mathbf{e}_{\|}, \mathbf{e}_{\perp}$, and $\mathbf{e}_{k}$ form a right-handed system congruent to $\mathbf{e}_{x}, \mathbf{e}_{y}$, and $\mathbf{e}_{z}$. The stated corollary follows upon setting $\rho_{q 0}(\mathbf{K})= \pm i$ and applying (22a) and (18).

\footnotetext{
${ }^{8}$ See, e.g., Beckmann [39] or Hollis et al. [40]. For optical terminology (which differs) see, e.g., Born and Wolf [33, p. 27].
} 


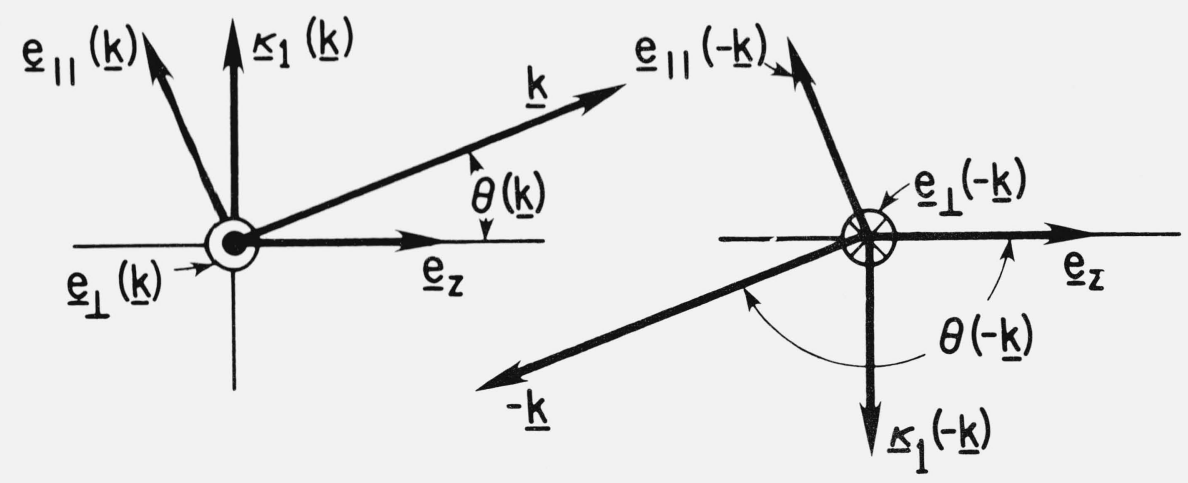

FIGURE 6. Unit vectors in and perpendicular to the plane of $\mathbf{e}_{z}$ and $\mathbf{k}$ for $\pm \mathbf{k}, \mathbf{k}$ real.

\section{Scattering-Matrix Analysis of Coupled Antennas; General Solution for System 2-Port}

We consider a system consisting of a pair of antenna systems operating in a homogeneous, isotropic, dissipationless medium, as shown in the highly schematic figure 7 . We are primarily interested in this system as a transmission system, with one antenna transmitting and the other receiving. The complete treatment of a transmission system must include effects of scattering by both antennas, and thus automatically includes treatment of reflection systems, in which one antenna functions in both transmitting and receiving modes and the other antenna represents an arbitrary passive (linear) scattering object.

For the description of the antenna on the left in figure 7, we apply (1.3-12). In the problem of interest there are no waves incident from the left $\left(\hat{a}_{2}=0\right)$; the spectrum of waves going to the left, $\hat{b}_{2}$, is not involved in the process of solving the problem (but it is obtainable as a part of the solution). Thus (1.3-12) reduces essentially to

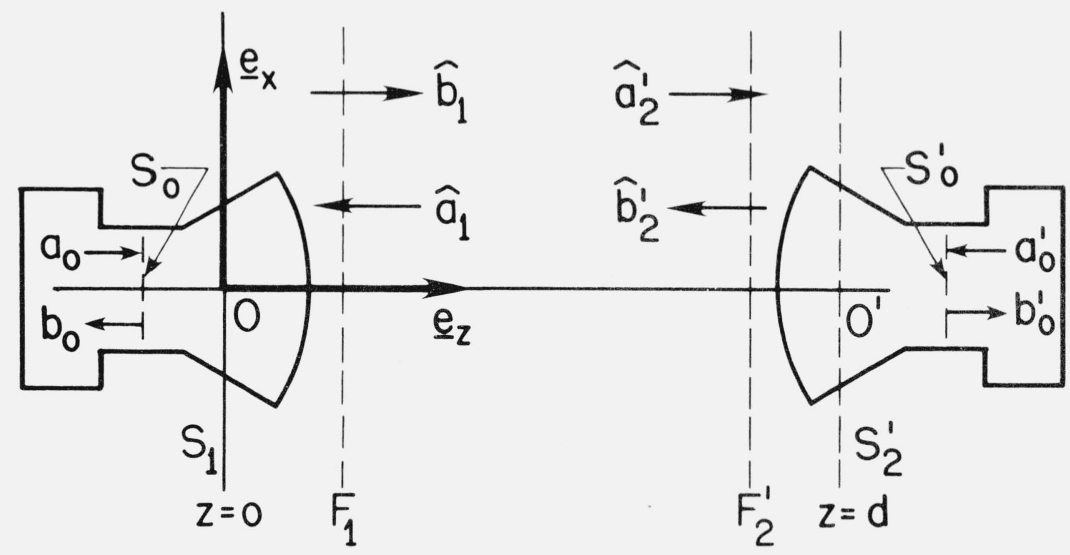

Figure 7. Transmission system-schematic.

$$
\begin{aligned}
& b_{0}=S_{00} a_{0}+\hat{S}_{01} \hat{a}_{1}, \\
& \hat{b}_{1}=\hat{S}_{10} a_{0}+\hat{S}_{11} \hat{a}_{1} .
\end{aligned}
$$

These equations are set up with reference to terminal surfaces $S_{0}$ and $S_{1}$, the latter being at $z=0$ in the coordinate system $0 x y z$. For the description of the antenna on the right, we again apply (1.3-12), using primes to distinguish quantities associated with this antenna. In the problem of 
interest there are no waves incident on the right side of this antenna $\left(\hat{a}_{1}^{\prime}=0\right)$ and the spectrum of waves going to the right, $\hat{b}_{1}^{\prime}$, is obtainable as a part of the solution. The needed scattering equations thus are

$$
\begin{aligned}
& b_{0}^{\prime}=S_{00}^{\prime} a_{0}^{\prime}+\hat{S}_{02}^{\prime} \hat{a}_{2}^{\prime}, \\
& \hat{b}_{2}^{\prime}=\hat{S}_{20}^{\prime} a_{0}^{\prime}+\hat{S}_{22}^{\prime} \hat{a}_{2}^{\prime} .
\end{aligned}
$$

For these equations the terminal surfaces are $S_{0}^{\prime}$ and $S_{2}^{\prime}$, the latter being $z=d$ in the coordinate system $0 x y z$.

The separation of the phase reference surfaces for the two antennas by the distance $d$ implies

$$
a_{2}^{\prime}(m, \mathbf{K})=b_{1}(m, \mathbf{K}) e^{i \gamma d}, \quad a_{1}(m, \mathbf{K})=b_{2}^{\prime}(m, \mathbf{K}) e^{i \gamma d} .
$$

Now, with respect to the transmission path as an element of the system, the set of incident waves is represented by $\hat{b}_{1}$ and $\hat{b}_{2}^{\prime}$ and the set of emergent waves by $\hat{a}_{1}$ and $\hat{a}_{2}^{\prime}$. Thus from (3), the matrix description of this element is

$$
\left(\begin{array}{l}
\hat{a}_{1} \\
\hat{a}_{2}^{\prime}
\end{array}\right)=\left(\begin{array}{ll}
\hat{0} & \hat{T} \\
\hat{T} & \hat{0}
\end{array}\right)\left(\begin{array}{c}
\hat{b}_{1} \\
\hat{b}_{2}^{\prime}
\end{array}\right)
$$

where the elements of $\hat{T}$ are $T(\mathbf{K} ; \mathbf{L})=\delta\left(k_{x}-l_{x}\right) \delta\left(k_{y}-l_{y}\right) e^{i \gamma(K) d}$. This is equivalent to the two separate transformations

$$
\hat{a}_{1}=\hat{T} \hat{b}_{2}^{\prime}, \hat{a}_{2}^{\prime}=\hat{T} \hat{b}_{1}
$$

We are now in a position to obtain a complete formal solution for the behavior of the transmission system under consideration. That is, we can obtain expressions for both $b_{0}$ and $b_{0}^{\prime}$, valid at arbitrary distances and including the effects of multiple reflections. (We can also formally determine the field in the transmission path.) We first consider transmission from left to right, assuming that the receiving antenna is terminated with a passive, reflectionless load. Using (2) and (5), we find

$$
\hat{a}_{1}=\hat{T} \hat{b}_{2}^{\prime}=\hat{T} \hat{S}_{22}^{\prime} \hat{a}_{2}^{\prime}=\hat{T} \hat{S}_{22}^{\prime} \hat{T} \hat{b}_{1}
$$

The operator $\hat{T} \hat{S}_{22}^{\prime} \hat{T}$ appearing here is the description of the receiving system, as a passive scattering object, referred to the reference plane $S_{1}$ of the transmitting antenna. Since this operator recurs frequently, we denote it briefly by $\hat{R}^{\prime}$. Substituting (6) in (1), we obtain

$$
\hat{b}_{1}=\hat{S}_{10} a_{0}+\hat{S}_{11} \hat{R}^{\prime} \hat{b}_{1}
$$

which (at least when written out more fully) is seen to be an integral equation determining $\hat{b}_{1}$. (It may be identified as an inhomogeneous, linear integral equation of the second kind.) The solution may be indicated formally by writing

$$
\hat{b}_{1}=\left(\hat{l}-\hat{S}_{11} \hat{R}^{\prime}\right)^{-1} \hat{S}_{10} a_{0} .
$$

This gives us the spectrum of outgoing waves in the transmission path; it includes both the simple plane waves and the evanescent waves. $\left(\hat{a}_{1}\right.$ is now determined by $(6) ; \mathbf{E}_{1 t}(\mathbf{r})$ and $\mathbf{H}_{1 t}(\mathbf{r})$ are determined by (1.2-8).) We may obtain a more explicit but still formal solution to the basic integral 
equation by the Liouville-Neumann method of successive substitutions. This leads to a representation of the inverse operator in (8) in a series of iterated operators,$^{9}$ so that

$$
\hat{b}_{1}=\left[\hat{1}+\hat{S_{11}} \hat{R}+\left(\hat{S_{11}} \hat{R}\right)^{2}+\ldots\right] \hat{S_{10}} a_{0} .
$$

The special virtue of this form is that the successive terms in the series correspond to successive round-trip multiple reflections between the transmitting and the receiving antennas. Of course, (9) is meaningful as an infinite series only if it converges in some useful sense. The domain of convergence will depend upon the "smallness" of the product $\hat{S}_{11} \hat{R}^{\prime}$, and it is worth noting that this product depends upon both $\hat{S}_{11}$ and $\hat{S}_{22}^{\prime}$ (as well as upon the distance between the transducers).

We complete this analysis by calculating the scattering matrix of the "system 2-port," which has its terminals at $S_{0}$ and $S_{0}^{\prime}$ and is defined by the equations

$$
\begin{aligned}
& b_{0}=M_{11} a_{0}+M_{12} a_{0}^{\prime}, \\
& b_{0}^{\prime}=M_{21} a_{0}+M_{22} a_{0}^{\prime},
\end{aligned}
$$

(The properties of a transmission system are often conveniently embodied in this form.) Inasmuch as we have made $a_{0}^{\prime}=0$, solving for $b_{0} / a_{0}$ and for $b_{0}^{\prime} / a_{0}$ yields directly

$$
\begin{aligned}
& M_{11}=S_{00}+\hat{S}_{01} \hat{R}^{\prime}\left(1-\hat{S}_{11} \hat{R}^{\prime}\right)^{-1} \hat{S}_{10}, \\
& M_{21}=\hat{S}_{02}^{\prime} \hat{T}\left(1-\hat{S}_{11} \hat{R}^{\prime}\right)^{-1} \hat{S}_{10} .
\end{aligned}
$$

A similar alternative solution with $a_{0}=0$ and $a_{0}^{\prime} \neq 0$ yields

$$
\begin{aligned}
& M_{22}=S_{00}^{\prime}+\hat{S}_{02}^{\prime} \hat{R}\left(1-\hat{S}_{22}^{\prime} \hat{R}\right)^{-1} \hat{S}_{20}^{\prime} \\
& M_{12}=\hat{S}_{01} \hat{T}\left(1-\hat{S}_{22}^{\prime} \hat{R}\right)^{-1} \hat{S}_{20}^{\prime},
\end{aligned}
$$

where $\hat{R} \equiv \hat{T} \hat{S}_{11} \hat{T}$. These formulas were first given in [41]; formally identical expressions are obtained in the electroacoustics case [3]. Their general significance was mentioned in the Introduction. Complete analytical solutions can be found in a highly specialized and idealized (but nevertheless interesting) class of problems; in one such solution the associated Liouville-Neumann series is incidentally found to converge or diverge according as $k d$ is greater or less than $0.87993310 \ldots$

In the measurement technique to be described in subsection 3.1 (but not that in 3.2 ) we assume that the effects of reflections between antennas have been minimized and may be neglected. When such reflections are omitted, (12) and (13) become $M_{21}=\hat{S}_{02}^{\prime} \hat{T} \hat{S}_{10}$ and $M_{22}=S_{00}^{\prime}$, respectively. If the (passive) termination on the receiving antenna has reflection coefficient $\Gamma_{l}$, we obtain from (10)

$$
b_{0}^{\prime}=F^{\prime} \hat{S}_{02}^{\prime} \hat{T} \hat{S}_{10} a_{0},
$$

where $F^{\prime}=\left(1-\Gamma_{L} S_{00}^{\prime}\right)^{-1}$. More explicitly, we have

$$
b_{0}^{\prime}=F^{\prime} a_{0} \int \sum S_{02}^{\prime}(m, \mathbf{K}) S_{10}(m, \mathbf{K}) e^{i \gamma d} d \mathbf{K}
$$

(Equation (15) is essentially a basic and simple case of eq (43) or (46) in [1].) The integral appearing in this equation is called the transmission integral, and the scalar product in the integrand,

${ }^{9}$ The operator expansion in $(9)$ is analogous to the finite-dimensional matrix expansion $(1-\mathrm{A})^{-1}=1+\mathrm{A}+\mathrm{A}^{2}+\mathrm{A}^{3}+\ldots \ldots$ which is valid if all the eigenvalues of $\mathrm{A}$ are less than unity in magnitude. 


$$
\sum_{m} S_{02}^{\prime}(m, \mathbf{K}) S_{10}(m, \mathbf{K}) \equiv \mathbf{S}_{02}^{\prime}(\mathbf{K}) \cdot \mathbf{S}_{10}(\mathbf{K})
$$

is called the coupling product. This marks the emergence of the central quantities involved in the antenna measurement techniques described here.

In order to establish some of the content of (15) (but not for present applications) we note that the well-known Friis transmission formula can be derived from the asymptotic form of the equation. The asymptotic form in question is

$$
b_{0}^{\prime} \sim-2 \pi i k F^{\prime} \mathbf{S}_{02}^{\prime}(0) \cdot \mathbf{S}_{10}(0) a_{0} e^{i k d} / d .
$$

(This is analytically a version of $(1.2-16 \mathrm{a})$ or $(1.2-16 \mathrm{~b})$ evaluated on-axis.) If we now calculate the ratio of the available power at the receiving antenna terminals to the net power input at the transmitting antenna terminals and use the Schwarz inequality, we obtain

$$
\frac{P_{A}^{\prime}}{P_{0}} \gtrless \frac{G_{1}(0) \sigma_{2}^{\prime}(0)}{4 \pi d^{2}}
$$

where $G_{1}(0)$ pertains to the transmitting antenna, $\sigma_{2}^{\prime}(0)$ pertains to the receiving antenna, and we have used (1.6-6, - 19). Equality in (18) holds for polarization match.

We mention one more important result, contained in (11). The first iterated integral in the Liouville-Neumann series for $M_{11}$ is the reflection integral,

$$
\Phi(d)=\int d \mathbf{K} e^{i \gamma(K) d} \mathbf{S}_{01}(\mathbf{K}) \cdot \int \mathbf{S}_{22}^{\prime}(\mathbf{K}, \mathbf{L}) \cdot \mathbf{S}_{10}(\mathbf{L}) e^{i \gamma(L) d} d \mathbf{L} .
$$

This is the simplest form of integral involving a scattering process. It can be interpreted as a monostatic radar equation, which, apart from multiple reflections between target and transceiver, is valid at arbitrary distances. Examples of the use of this equation may be found in [1]. In particular, upon setting $\mathbf{S}_{22}^{\prime}(\mathbf{K}, \mathbf{L})=-\mathbf{l} \delta(\mathbf{K}-\mathbf{L})$, where $\mathbf{l}$ denotes the transverse unit dyadic, one obtains the interferometer reflection-integral of reference [1].

\section{Determination of Coupling-Product Values}

\subsection{Deconvolution of Transverse Scanning Data; Application of Sampling Theorem}

We can now quite easily give the analytical basis for determining coupling-product values from transmission data taken in a transverse plane. Let the required relative transverse displacement between the transmitting and the receiving antennas be denoted by a transverse displacement $\mathbf{P}=x \mathbf{e}_{x}+y \mathbf{e}_{y}$ of the receiving antenna (fig. 8). By considering the phase $\mathbf{k} \cdot \mathbf{r}\left(\mathbf{r}=\mathbf{P}+d \mathbf{e}_{z}\right)$ of the waves in the spectrum incident on the reference plane $S_{2}^{\prime}$ of the receiving antenna, we see that the process of displacement simply introduces the factor $\exp (i \mathbf{K} \cdot \mathbf{P})$ in the integrand of $(2-15)$. The expression for the received signal becomes

$$
b_{0}^{\prime}(\mathbf{P})=a_{0} F^{\prime} \int e^{i \kappa \cdot \mathbf{P}} \mathbf{S}_{02}^{\prime}(\mathbf{K}) \cdot \mathbf{S}_{10}(\mathbf{K}) e^{i \gamma d} d \mathbf{K} .
$$

Note that the quantity $b_{0}^{\prime}(\mathbf{P})$ is what is observed in the measurement process; it may or may not be simply related to $\mathbf{E}$ at the point $(\mathbf{P}, d)$. Inasmuch as (1) represents a Fourier integral transformation, its inversion is immediate: We write

$$
\mathbf{S}_{02}^{\prime}(\mathbf{K}) \cdot \mathbf{S}_{10}(\mathbf{K})=D(\mathbf{K})
$$

where $D(\mathbf{K})$ is here an abbreviation for the determinate function of $\mathbf{K}$ given by 


$$
D(\mathbf{K}) \equiv \frac{e^{-i \gamma d}}{4 \pi^{2} F^{\prime} a_{0}} \int b_{0}^{\prime}(\mathbf{P}) e^{-i \mathbf{K} \cdot \mathbf{P}} d \mathbf{P} .
$$

The inversion of (1) is appropriately termed deconvolution (each of the factors $\mathbf{S}_{02}^{\prime}$ and $\mathbf{S}_{10}$ can be interpreted as Fourier transforms of certain physical fields). The term distinguishes the inversion of (1) from the inversion of the simpler equation (1.3-6) representing the Fourier-transform definition of $\mathbf{S}_{q 0}$.

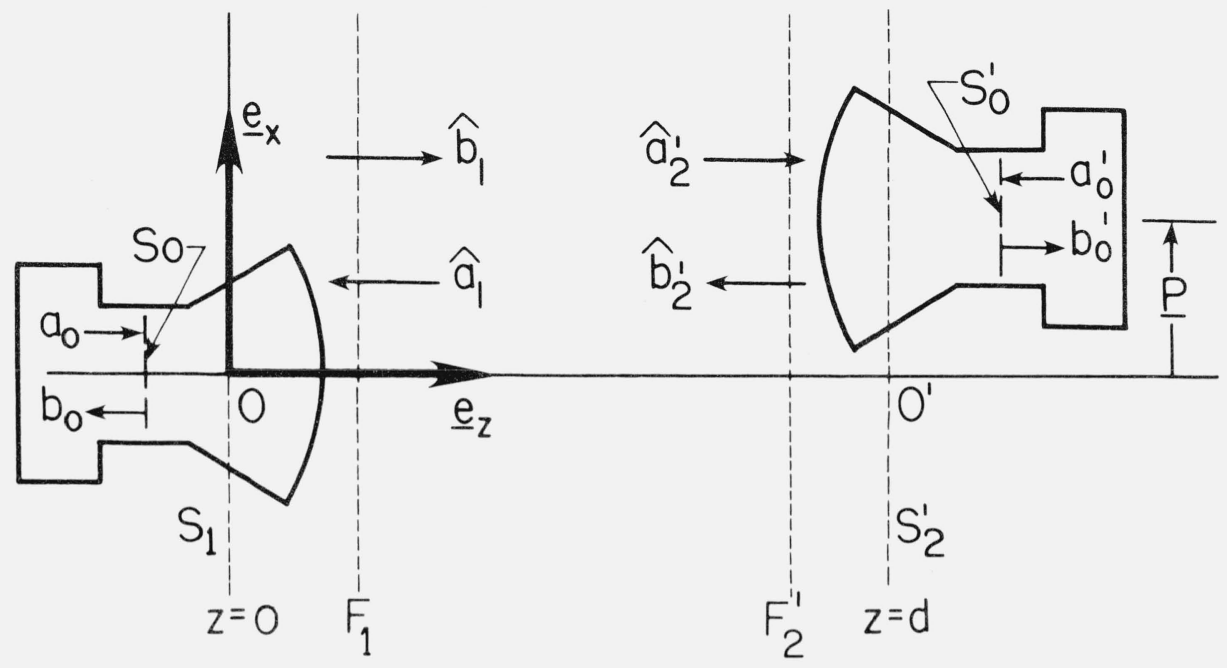

Figure 8. Arrangement for transverse scanning: $d$ is fixed, $\mathbf{P}$ is variable.

The right-hand side of (3) is in fact determined (up to a phase factor) by the following measurable quantities: the distance $d$ between the reference planes $S_{1}$ and $S_{2}^{\prime}$; the reflection coefficients $\Gamma_{L}$ and $S_{00}^{\prime}$; and the magnitude and relative phase of $b_{0}^{\prime}(\mathbf{P}) / a_{0}$ as a function of $\mathbf{P}$. (For convenience in measurement $b_{0}^{\prime}(\mathbf{P})$ may be normalized in two stages, indicated by the expression

$$
\frac{b_{0}^{\prime}(\mathbf{P})}{a_{0}}=\frac{b_{0}^{\prime}\left(\mathbf{P}_{0}\right)}{a_{0}} \cdot \frac{b_{0}^{\prime}(\mathbf{P})}{b_{0}^{\prime}\left(\mathbf{P}_{0}\right)},
$$

where $\mathbf{P}_{0}$ is a selected fixed point.)

An instructive hypothetical case in which our analysis correctly shows that no probe correction, other than a known constant multiplier, would be needed occurs when the receiving antenna is considered to be an ideal electric-field probe. Such a probe can be described and treated analytically as an elementary electric dipole antenna, assumed lossless and reciprocal. For such a probe it can be shown that the receiving response is $b_{0}^{\prime}(\mathbf{P})=C \mathbf{E}(\mathbf{P}) \cdot \mathbf{e}_{p}$, where $\mathbf{e}_{p}$ is a unit vector giving the orientation of the dipole and the absolute value of the constant $C$ can be calculated. Evidently the assumed use of an ideal probe oriented in the $x$-direction, say, in (3), would effectively reduce (3) to the $\mathbf{e}_{x}$-component of (1.3-6).

As (1) shows, planar scanning (in the absence of multiple reflections) can be interpreted rigorously as a spatially invariant, linear, filtering process in the two-dimensional wavenumber domain (for filtering concepts see e.g., [53]). The concept of the action of a receiving antenna as a filter has been noted, e.g., by Brown [19], apparently with non-planar scanning in mind. The processes are substantially different in the planar and the non-planar cases, as becomes clearly evident upon consideration of the action of a highly directive receiving antenna. Nevertheless, the ability to account fully for the probe characteristics in the planar case suggests the use of a well-charac- 
terized "large" probe to reduce data taking and processing effort. Measurements at NBS using such a probe have produced the expected beneficial results. The filtering process has also been studied by Joy and Paris [10] using digital filtering (after data taking) to simulate probe characteristics.

Clearly the utility of the result (3) depends upon one's ability to evaluate the transform of the empirically observed $b_{0}^{\prime}(\mathbf{P})$. Both least-square fitting and a two-dimensional form of the sampling theorem have been successfully used to evaluate the required transform of $b_{0}^{\prime}(\mathbf{P})$ from data taken at the points of a rectangular lattice in the measurement plane [2,8]. The application of the sampling theorem has become the method of choice, mainly because of the greater ease of computation, and will be described very briefly here.

The essential requirement of the sampling theorem is that the function to be sampled be representable as the Fourier transform of a band-limited function. That $b_{0}^{\prime}(\mathbf{P})$ virtually fulfills this condition may be seen from (1): a band-limit $K_{B}$ somewhat greater than $k$, and a distance $d$, may be chosen so that evanescent waves for all $K>K_{B}$ are assuredly virtually zero in the measurement plane (e.g., with $K_{B}=1.05 k$ and $d=15 \lambda$, attenuation at the band limit is approximately $260 \mathrm{~dB}$ ). Bandlimiting within a smaller spectral region may result from the behavior of the product $\mathbf{S}_{02}^{\prime}(\mathbf{K})$. $\mathbf{S}_{10}(\mathbf{K})$ in individual cases (an early study is reported in [8]). If we assign band limits $k_{1} \equiv \pm 2 \pi / \lambda_{1}$ and $k_{2} \equiv \pm 2 \pi / \lambda_{2}$ for $k_{x}$ and $k_{y}$, respectively, a straightforward generalization of the usual onedimensional theory, given in appendix $\mathrm{C}$, leads to

$$
D(\mathbf{K})=\frac{e^{-i \gamma d}}{4 k_{1} k_{2} F^{\prime} a_{0}} \sum_{r, s} b_{0}^{\prime}\left(\mathbf{P}_{r s}\right) e^{-i \mathbf{K} \cdot \mathbf{P} r s}
$$

The vectors $\mathbf{P}_{r s}=\frac{1}{2} r \lambda_{1} \mathbf{e}_{x}+\frac{1}{2} s \lambda_{2} \mathbf{e}_{y}$ (with $r, s=\ldots .-1,0,1,2, \ldots$.) define the measurement lattice, the quantities $b_{0}^{\prime}\left(\mathbf{P}_{r s}\right)$ are the (complex) values of probe output directly observed at the points of the lattice, and the summation goes over the points of the lattice. According to the sampling theorem, (4) is mathematically exact; that is, if the data [the $\left.b_{0}^{\prime}\left(\mathbf{P}_{r s}\right)\right]$ were complete and exact, the result would be exact. (Although (4) is exact, it is not the "best possible" result; more advanced theory [42] shows that a rhomboidal lattice would be somewhat more efficient than the rectangular lattice.) The theorem requires an infinite sum, but in the applications thus far we have found that not even all values measurable above noise are needed.

An important feature of (4) is that the highly efficient algorithm known as the "fast Fourier transform" is rigorously applicable to evaluate the sum.

\subsection{Received Signal as a Function of Distance and the Extrapolation Technique}

The extrapolation technique was introduced by Wacker and Bowman, has been described very briefly by Newell and Kerns [9], and more fully, in an experimentally oriented paper, by Newell et al. [11]. The theory and numerical techniques were developed by Wacker [6]. The papers mentioned should be consulted for details; in the following paragraphs we give only a brief account of the main ideas and equations involved.

The extrapolation technique requires that one observe $b_{0}^{\prime} / a_{0}$ as a function of antenna separation distance $d$, which is precisely defined by the choice of reference surfaces $S_{1}$ and $S_{2}^{\prime}$ associated with the respective antennas (fig. 7).

From (2-10) for the system 2-port, we obtain the expression

$$
b_{0}^{\prime}=a_{0} M_{21} /\left(1-M_{22} \Gamma_{L}\right),
$$

which is a precise and complete version of (2-15). Expressions for the elements $M_{21}$ and $M_{22}$ are given in $(2-12,-13)$, from which, by a rather lengthy process, one finds for (1) as a function of $d$ a series representation of the form 


$$
b_{0}^{\prime}=a_{0} F^{\prime} \sum_{p=0}^{\infty} \frac{\exp [i(2 p+1) k d]}{d^{2 p+1}} \sum_{q=0}^{\infty} A_{p q} d^{-q} .
$$

We observe that the subseries of terms with a given $p$ can be interpreted as the contribution of energy which has experienced $2 p$ reflections or made $2 p+1$ transits between antennas. In particular, the subseries with $p=0$ involves no reflections and is the expansion of the transmission integral $(2-15)$ :

$$
\Psi(d)=\frac{e^{i k d}}{d}\left(A_{00}+A_{01} d^{-1}+A_{02} d^{-2}+\ldots\right) .
$$

It is of considerable analytical interest that this series is not merely asymptotic but actually convergent for sufficiently large $d$, under the main hypothesis that the two antennas involved be of finite size. If $d$ is measured between centers of spheres, of radii $r$ and $r^{\prime}$, each circumscribing one of the antennas, then $d>d_{0}=r+r^{\prime}$ is sufficient. A generally sharper, but more complicated, prescription for $d_{0}$ can be given $[6,7]$.

By comparison of $(3)$ and $(2-17)$ we see that

$$
A_{00}=-2 \pi i h_{\mathbf{S}}^{\prime}(0) \cdot \mathbf{S}_{10}(0) .
$$

Hence determination of the leading coefficient in (2) is tantamount to the determination of the (onaxis) value of the spectral coupling product. The basic idea of what we may call the conventional measurement method is simply to have $d$ large enough to make other terms negligible compared to the leading term of the series. The basic idea of the extrapolation technique is to observe $b_{0}^{\prime}$ as a function of $d$ and to fit this function with as many terms of (2) as may be significant, and so to determine a good value for $A_{00}$ in particular. This enables one to cope with proximity effects and with multiple reflections between antennas.

\section{Utilization of Coupling-Product Data}

\subsection{One Unknown Antenna (Transmitting or Receiving)}

In this subsection we provide basic equations for the use of coupling-product data in a basic antenna measurement situation: the measurement of an unknown antenna (transmitting or receiving) with the requisite known antennas or antenna. Some of the concepts and notation established will be used in the next two subsections (which provide a partial answer to the question, "How does one obtain the first known antenna?").

In this subsection "nothing more" than the algebra of two (complex) equations in two (complex) unknowns is involved. The only likely case involving non-uniqueness and compatibility conditions is discussed. (In the following subsections the linear equations have to be reinterpreted as quadratics.)

It will suffice to consider only the case in which the unknown antenna is transmitting; the discussion of the other case would, of course, be analytically very similar.

We do not make simplifying a priori assumptions concerning symmetry or polarization (or other) characteristics of the antenna to be measured. Quite generally, then, we require measurements to be made with (at least in effect) two receiving antennas, A and B, having suitable known receiving characteristics, $\mathbf{A}_{02}(\mathbf{K})$ and $\mathbf{B}_{02}(\mathbf{K})$. From such measurements, the values of the coupling products

$$
\begin{aligned}
& \mathbf{A}_{02}(\mathbf{K}) \cdot \mathbf{S}_{10}(\mathbf{K})=D_{A}(\mathbf{K}), \\
& \mathbf{B}_{02}(\mathbf{K}) \cdot \mathbf{S}_{10}(\mathbf{K})=D_{B}(\mathbf{K})
\end{aligned}
$$


are to be determined for the desired values of $\mathbf{K}$. For each chosen, fixed value of the parameter $\mathbf{K}$, we have two (complex) equations for the two (complex) components of $\mathbf{S}_{10}(\mathbf{K})$.

Complete solvability of (1) requires that the vectors $\mathbf{A}_{02}$ and $\mathbf{B}_{02}$ be linearly independent at the value of $\mathbf{K}$ considered. A measure of the linear independence of the two vectors is given by the following expression for the normalized squared-magnitude of the determinant of (1):

$$
\frac{|\Delta|^{2}}{|\mathbf{A}|^{2}|\mathbf{B}|^{2}}=1-\frac{|\mathbf{A} \cdot \overline{\mathbf{B}}|^{2}}{|\mathbf{A}|^{2}|\mathbf{B}|^{2}}
$$

As the Schwarz inequality shows, this quantity ranges from the value zero when $\mathbf{B}_{02}$ is proportional to $\mathbf{A}_{02}$ to a maximum of unity when $\overline{\mathbf{B}}_{02} \cdot \mathbf{A}_{02}$ vanishes. In other words, power orthogonality represents the extreme case of linear independence. Examples are linear polarizations at right angles and left- and right-circular polarizations.

An explicit, coordinate-free solution of (1) may be obtained with the use of the set of vectors reciprocal to $\mathbf{A}_{02}$ and $\mathbf{B}_{02}$. The reciprocal set $\boldsymbol{\alpha}, \boldsymbol{\beta}$ is defined by

$$
\boldsymbol{\alpha} \cdot \mathbf{A}_{02}=1, \quad \boldsymbol{\alpha} \cdot \mathbf{B}_{02}=\boldsymbol{\beta} \cdot \mathbf{A}_{02}=0, \quad \boldsymbol{\beta} \cdot \mathbf{B}_{02}=1
$$

(assuming the required linear independence), and

$$
\mathbf{S}_{10}=D_{A} \boldsymbol{\alpha}+D_{B} \boldsymbol{\beta}
$$

as is easily verified. The algebra is summed up in the statement that $D_{A}$ and $D_{B}$ are the covariant components of $\mathbf{S}_{10}$ with respect to $\mathbf{A}_{02}$ and $\mathbf{B}_{02}$ as base vectors.

In much of what follows the use of $x, y$ components relative to the fixed basis $\mathbf{e}_{x}, \mathbf{e}_{y}-$ rather than the 1,2 components relative to the variable unit vectors $\kappa_{1}, \kappa_{2}-$ is indicated. The consideration of geometric rotations and symmetries is appreciably complicated by the dependence of the $\boldsymbol{\kappa}$ 's upon K. Also, the use of $\mathbf{e}_{x}, \mathbf{e}_{y}$ automatically takes care of the matter of defining the $\boldsymbol{\kappa}$ 's onaxis. The required coordinate transformations will be governed by the relationship of the unit vectors

$$
\boldsymbol{\kappa}_{1}=c \mathbf{e}_{x}+s \mathbf{e}_{y}, \quad \boldsymbol{\kappa}_{2}=-s \mathbf{e}_{x}+c \mathbf{e}_{y},
$$

where $c \equiv \cos \phi=k_{x} / K$ and $s \equiv \sin \phi=h_{\text {iy }} / K$.

We introduce the abbreviations

$$
A_{x}=A_{02 x}(\mathbf{K}), \quad B_{x}=B_{02 x}(\mathbf{K}), \quad S_{x}=S_{10 x}(\mathbf{K}), \quad(x=x, y)
$$

and write (1) in component form

$$
\begin{aligned}
& A_{x} S_{x}+A_{y} S_{y}=D_{A}, \\
& B_{x} S_{x}+B_{y} S_{y}=D_{B} .
\end{aligned}
$$

Instead of using two intrinsically different antennas $\mathrm{A}$ and $\mathrm{B}$, in many cases it may be possible and convenient to use one antenna in two orientations, differing by rotation around the $z$ axis by 90 degrees, say in the direction of $x$ to $y$. If antenna A is so used and so rotated, we obtain for the equivalent of antenna $B$

$$
\begin{aligned}
& B_{02 x}\left(k_{x}, k_{y}\right)=-A_{02 y}\left(k_{y},-k_{x}\right) \\
& B_{02 y}\left(k_{x}, k_{y}\right)=A_{02 x}\left(k_{y},-k_{x}\right) .
\end{aligned}
$$


These equations express the rotation of the vector field $\mathbf{A}_{02}$ corresponding to the rotation of the antenna that it describes. ${ }^{10}$ They lead to a modified version of (6), which we shall discuss in the particularly interesting case of evaluation on-axis $(\mathbf{K}=0)$. If we let $A_{x}^{0}=A_{02 x}(0), S_{x}^{0}=S_{10 x}(0)$, etc., the modified form of (6) is

$$
\begin{aligned}
A_{x}^{0} S_{x}^{0}+A_{y}^{0} S_{y}^{0} & =D_{A}(0), \\
-A_{y}^{0} S_{x}^{0}+A_{x}^{0} S_{y}^{0} & =D_{B}(0) .
\end{aligned}
$$

The determinant of the system (8) vanishes if and only if $A_{x}^{0}= \pm i A_{y}^{0}$; that is, the determinant vanishes if and only if the response characteristic $\mathbf{A}_{02}$ is "circularly polarized" at the point $\mathbf{K}=0$. This gives us a hint as to the special advantages of the use of circular polarization components for equations of the above form. We introduce circular polarization components for on-axis quantities in the following manner: For the transmitting characteristic

$$
\mathbf{S}_{10}(0)=S_{+}^{0} \mathbf{e}_{+}+S_{-}^{0} \mathbf{e}_{-}
$$

and for the receiving characteristic

$$
\mathbf{A}_{02}(0)=A_{+}^{0} \overline{\mathbf{e}}_{+}+A_{-}^{0} \overline{\mathbf{e}}_{-},
$$

where the superposed bar denotes the complex conjugate (as usual) and

$$
\mathbf{e}_{+} \equiv\left(\mathbf{e}_{x}+i \mathbf{e}_{y}\right) / \sqrt{2}, \quad \mathbf{e}_{-} \equiv\left(\mathbf{e}_{x}-i \mathbf{e}_{y}\right) / \sqrt{2}
$$

(The ordered pair of vectors $\overline{\mathbf{e}}_{+}, \overline{\mathbf{e}}_{-}$in $(9 \mathrm{~b})$ is reciprocal to the ordered pair $\mathbf{e}_{+}, \mathbf{e}_{-}$in (9a).) The formulas for transformation to circular components are thus determined as

$$
\begin{gathered}
S_{x}^{0}=\left(S_{+}^{0}+S_{-}^{0}\right) / \sqrt{2}, \quad S_{y}^{0}=i\left(S_{+}^{0}-S_{-}^{0}\right) / \sqrt{2}, \\
A_{x}^{0}=\left(A_{+}^{0}+A_{-}^{0}\right) / \sqrt{2}, \quad A_{y}^{0}=-i\left(A_{+}^{0}-A_{-}^{0}\right) / \sqrt{2} .
\end{gathered}
$$

The scalar products in (8) become

$$
\begin{gathered}
A_{+}^{0} S_{+}^{0}+A_{-}^{0} S_{-}^{0}=D_{A}(0), \\
i A_{+}^{0} S_{+}^{0}-i A_{-}^{0} S_{-}^{0}=D_{B}(0) .
\end{gathered}
$$

As is apparent, we have chosen the notation so that $A_{+}$and $A_{-}$represent receptivities to the correspondingly labeled circular components of $\mathbf{S}_{10}$. The coupling is of course consistent with the polarization matching theorem (1.6-18). Equations (11) show clearly that if the receiving antenna were to respond to only one circular component of polarization, then that component, but not the other, could still be measured using one or the other of the two equations. If both equations were to be used, the compatibility condition (required by the vanishing of the determinant) should be satisfied within experimental error.

\subsection{Generalized Two-Identical-Antenna Techniques}

The technique to be described is formulated for nonreciprocal antennas, assuming that one possesses the adjoint of the antenna to be measured. If the antenna to be measured is reciprocal

${ }^{10}$ Concepts, analytical tools, and notation for the rotation of vector and tensor fields are discussed on p. 272 of [43]. 
( $\equiv$ self-adjoint), then the assumption is that one possesses duplicate antennas. ${ }^{11}$ This is no doubt the more likely case but the more general formulation can be given with essentially no extra algebraic complication. Under the main assumption we are assured that the receiving characteristic of one antenna will be related to the transmitting characteristic of the other by reciprocity relations whether or not the antennas are reciprocal.

Granted the assumption of duplicate or mutually adjoint antennas, no additional assumptions are required to permit formulation and solution of equations for on-axis gain and polarization characteristics of both antennas. Additional a priori information, ordinarily qualitative, is required only for resolution of square-root sign ambiguities.

Certain commonly occurring types of symmetry permit one additionally to obtain solutions for off-axis values of gain and polarization.

For definiteness we assume transmission from left to right (as usual); limit the discussion to the determination of the right-side characteristics of the two (in general distinct) antennas labeled $S$ and $S^{a}$, say; and choose to formulate equations for the direct determination of transmitting characteristics, leaving receiving characteristics to be determined by reciprocity. Under these ground rules, the remaining problem consists of at most two parts:

(a) Transmit from $S$ to $S^{a}$; formulate equations for $\mathbf{S}_{10}$, find $\mathbf{S}_{o 1}^{a}$ by reciprocity;

(b) Transmit from $S^{a}$ to $S$; formulate equations for $\mathbf{S}_{10}^{a}$, find $\mathbf{S}_{01}$ by reciprocity.

If reciprocity (in the ordinary sense) applies, the superscript " $a$ " is without effect and may be omitted; the two cases reduce to one.

Inasmuch as the algebraic problem is in all cases substantially identical, it will be sufficient to consider only case (a) explicitly.

Both antennas are initially to be described in the same orientation and position relative to the fixed coordinate system $0 x y z$. When one of the antennas is placed and oriented to serve in reception, its description relative to fixed coordinates will be changed accordingly. Indeed, the phase factor $\exp (i \gamma d)$ introduced by the axial translation can be regarded either as a modification of the receiving characteristic of the receiving antenna or as a property of the transmission path. We make the latter point of view explicit by referring the description of the receiving antenna to the shifted coordinate system $0^{\prime} x y z^{\prime}$, where $0^{\prime}$ is at point $(0,0, d)$ in the original system $0 x y z$ (fig. 7).

We shall need to consider the receiving antenna in two receiving orientations, differing by a 90 degree rotation around the $z$-axis. The operative characteristics of the receiving antenna in these two orientations will be distinguished by single and double primes.

Let the adjoint antenna be rotated into the first receiving orientation. This requires 180 degrees rotation around a transverse axis, say the $y$-axis. The operative receiving characteristic of the rotated antenna is then

$$
\mathbf{S}_{02}^{a^{\prime}}(\mathbf{K})=P_{y 2} \mathbf{S}_{01}^{a}(\mathbf{K}),
$$

where $P_{y 2}$ is a notation for the transformation ( of $\mathbf{S}_{01}^{a}$ ) produced by the prescribed rotation. Now the transformed function $P_{y 2} \mathbf{S}_{01}^{a}$ is related by reciprocity, (1.5-4), to the similarly transformed function $P_{y 2} \mathbf{S}_{10}$. Thus, if the reciprocity relation is written in vector form, we have

$$
\eta_{0} \mathbf{S}_{02}^{a^{\prime}}(\mathbf{K})=\boldsymbol{\eta} \cdot P_{y 2} \mathbf{S}_{10}(-\mathbf{K})
$$

and if it is written in $x, y$ component form we have

11 The idea of using a reflecting surface or mirror to produce an image antenna is not fully applicable, even if the antenna is reciprocal: Coupling-product data can be obtained by extrapolation or by conventional techniques. but not by transverse scanning. The coupling-product equations can be formulated and solved for on-axis values provided on-axis polarization is known and not circular. It should be noted that the image antenna, being a mirror image, cannot in general be considere an identical antenna even with a perfectly reflecting surface of infinite area. 


$$
\boldsymbol{\eta}_{0}\left(\begin{array}{c}
S_{02 x}^{a^{\prime}(\mathbf{K})} \\
S_{02 y}^{a^{\prime}(\mathbf{K})}
\end{array}\right)=\left(\begin{array}{cc}
\eta_{1} c^{2}+\eta_{2} s^{2} & \left(\eta_{1}-\eta_{2}\right) c s \\
\left(\eta_{1}-\eta_{2}\right) c s & \eta_{1} s^{2}+\eta_{2} c^{2}
\end{array}\right)\left(\begin{array}{l}
-S_{10 x}\left(k_{i x},-l_{i y}\right) \\
S_{10 y}\left(k_{x},-k_{y}\right)
\end{array}\right)
$$

Here the square matrix is determined by (4.1-4) and the column matrix on the right contains the $x, y$ components of $P_{y 2} \mathbf{S}_{10}(-\mathbf{K})$. We may now evaluate the coupling product $\mathbf{S}_{10}(\mathbf{K}) \cdot \mathbf{S}_{02}^{a^{\prime}}(\mathbf{K})$ $\equiv D^{\prime}(\mathbf{K})$. Some degree of abbreviation is indispensable; we use

$$
S_{x}=S_{10 x}(\mathbf{K}), \quad S_{y}=S_{10 y}(\mathbf{K}), \quad S_{x}^{v}=S_{10 x}\left(k_{x},-k_{y}\right), \quad S_{y}^{v}=S_{10 y}\left(k_{i x},-k_{y}\right),
$$

and obtain

$-\left(\eta_{1} c^{2}+\eta_{2} s^{2}\right) S_{x} S_{x}^{v}+\left(\eta_{1}-\eta_{2}\right) s c S_{x} S_{y}^{v}+$

$$
-\left(\eta_{1}-\eta_{2}\right) s c S_{y} S_{x}^{v}+\left(\eta_{1} s^{2}+\eta_{2} c^{2}\right) S_{y} S_{y}^{v}=\eta_{0} D^{\prime}(\mathbf{K}) .
$$

This is the first of the desired "measurement equations" relating the mathematical expression of the coupling product to its empirically determined values. It is interesting that the expression is invariant with respect to the interchange of $k_{i y}$ and $-k_{y}$; the empirical $D^{\prime}(\mathbf{K})$ should also have this symmetry. Further, since $D^{\prime}(\mathbf{K})$ is related to $b_{0}^{\prime}(\mathbf{P})$ by $(3.1-1)$, the $b_{0}^{\prime}(\mathbf{P})$ data should have the corresponding property of invariance with respect to the interchange of $y$ and $-y$. These general constraints should be experimentally useful.

To obtain the second measurement equation, we rotate the receiving antenna, as described by (1), 90 degrees around the $z$-axis in the direction $x$ to $y$ (cf. (4.1-7)). Using the notation $P_{z 4}$ for this rotation and applying the reciprocity relation, as in (2), we obtain

$$
\eta_{0} \mathbf{S}_{02}^{a^{\prime \prime}}(\mathbf{K})=\eta \cdot P_{z 4} P_{y^{2}} \mathbf{S}_{10}(-\mathbf{K})
$$

In $x, y$ component form this is

$$
\eta_{0}\left(\begin{array}{c}
S_{02 x}^{a^{\prime \prime}}(\mathbf{K}) \\
S_{02 y}^{a^{\prime \prime}}(\mathbf{K})
\end{array}\right)=\left(\begin{array}{cc}
\eta_{1} c^{2}+\eta_{2} s^{2} & \left(\eta_{1}-\eta_{2}\right) s c \\
\left(\eta_{1}-\eta_{2}\right) s c & \eta_{1} s^{2}+\eta_{2} c^{2}
\end{array}\right)\left(\begin{array}{l}
-S_{10 y}\left(k_{i y}, h_{i x}\right) \\
-S_{10 x}\left(h_{i y}, h_{i x}\right)
\end{array}\right)
$$

Using the abbreviations

$$
S_{x}^{d}=S_{10 x}\left(k_{y}, k_{i x}\right), \quad S_{y}^{d}=S_{10 y}\left(k_{y}, k_{x}\right),
$$

as well as $S_{x}$ and $S_{y}$ in (4), we find for the coupling product $S_{10}(\mathbf{K}) \cdot \mathbf{S}_{02}^{a^{\prime \prime}}(\mathbf{K}) \equiv D^{\prime \prime}(\mathbf{K})$ the expression

$$
\begin{aligned}
-\left(\eta_{1} c^{2}+\eta_{2} s^{2}\right) S_{x} S_{y}^{d}-\left(\eta_{1}-\eta_{2}\right) s c S_{x} S_{x}^{d} & + \\
& -\left(\eta_{1}-\eta_{2}\right) s c S_{y} S_{y}^{d}-\left(\eta_{1} s^{2}+\eta_{2} c^{2}\right) S_{y} S_{x}^{d}=\eta_{0} D^{\prime \prime}(\mathbf{K}) .
\end{aligned}
$$

In this case the coupling product is invariant with respect to the interchange of $h_{x}$ and $h_{i y}$, and again this constraint should be experimentally useful.

In the remainder of this subsection we discuss briefly some conditions and methods for determining components of $\mathbf{S}_{10}$ from (5) and (9), assuming that the $D$ 's are given for the values of $\mathbf{K}$ of interest. When evaluated for $\mathbf{K} \neq 0$, the six quantities $S_{x}, S_{y}, S_{x}^{v}, S_{y}^{v}, S_{x}^{d}$, and $S_{y}^{d}$ appearing 
in these two equations are in general distinct and unknown; obviously, some specialization or additional data are required. We consider two cases: evaluation on-axis, and a simple type of symmetry.

(i) Evaluation on axis-Evaluated on-axis, the six unknowns reduce to two

$$
S_{x}^{0}=S_{10 x}(0), \quad S_{y}^{0}=S_{10 y}(0),
$$

and the coupling-product equations reduce to

$$
\begin{gathered}
-\left(S_{x}^{0}\right)^{2}+\left(S_{y}^{0}\right)^{2}=D^{\prime}(0) \eta_{0} / Y_{0}, \\
-2 S_{x}^{0} S_{y}^{0}=D^{\prime \prime}(0) \eta_{0} / Y_{0} .
\end{gathered}
$$

(The key to this reduction is the observation that $Y_{0}$ is the common value of $\eta_{1}$ and $\eta_{2}$ on-axis.) Subcases under this case occur if the polarization on-axis is considered known. One or the other of (11) will suffice, no matter what that polarization may be.

Suppose, for example, that $\rho=S_{y}^{0} / S_{x}^{0}$ is considered known and not equal to \pm 1 ; then from (11a) we may obtain

$$
S_{x}^{0}=\left(\frac{\eta_{0} D^{\prime}(0)}{Y_{0}\left(\rho^{2}-1\right)}\right)^{1 / 2}
$$

This, together with $S_{y}^{0}=\rho S_{x}^{0}$, gives us the on-axis pattern vector in terms of $D^{\prime}(0)$ (up to a sign). From the expression (1.6-6) for power gain we find

$$
G_{1}(0)=\frac{4 \pi k^{2}\left(|\rho|^{2}+1\right)\left|D^{\prime}(0)\right|}{\left(1-\left|S_{00}\right|^{2}\right)\left|\rho^{2}-1\right|} .
$$

We can obtain an interesting form for this result by expressing $D^{\prime}(0)$ in terms of the integral of $b_{0}^{\prime}(\mathbf{P})$, as in the deconvolution relation, (3.1-3). Thus,

$$
G_{1}(0)=\frac{k_{i}^{2}}{\pi} \frac{\left|1-\Gamma_{l}^{\prime} S_{00}^{\prime}\right|}{1-\left|S_{00}\right|^{2}} \frac{|\rho|^{2}+1}{\left|\rho^{2}-1\right|} \frac{1}{\left|a_{0}\right|}\left|\int b_{0}^{\prime}(\mathbf{P}) d \mathbf{P}\right|
$$

(This result, as well as the version of it with $\rho=0$, has been presented previously [41, 44].) The essential simplicity of the result is somewhat obscured by the presence of the mismatch factors. If we assume a polarization match $(\rho=0, \pm i$, or $\infty)$, a conjugate impedance match $\left(\Gamma_{L}^{\prime}=\bar{S}_{00}^{\prime}\right)$, and $S_{00}=S_{00}^{\prime}$ (as appropriate for mutually adjoint or for identical antennas), we have

$$
G_{1}(0)=\frac{k_{i}^{2}}{\pi} \cdot \frac{1}{\left|a_{0}\right|}\left|\int b_{0}^{\prime}(\mathbf{P}) d P\right|
$$

For the effective area, using $(1.6-21 b)$, we obtain the remarkably simple expression

$$
\sigma_{1}^{a}(0)=\frac{1}{\left|a_{0}\right|}\left|\int b_{0}^{\prime}(\mathbf{P}) d \mathbf{P}\right|
$$

Equation (13) has been successfully applied experimentally [8], and (15) has been tested analytically in a special case. In the analytical test the two identical antennas were taken to be $x$-oriented elementary electric-dipole antennas, assumed lossless and reciprocal. In this case one does indeed obtain the expected result $\sigma_{1}(0)=3 \lambda^{2} /(8 \pi)$.

More generally, the polarization is not known and it is necessary to solve (11) as simultaneous quadratics. A solution in terms of circular polarization components is convenient and useful. 
Using the definitions in (4.1-9a), one obtains

$$
\begin{aligned}
& \left(S_{+}^{0}\right)^{2}=-\frac{\eta_{0}}{2 Y_{0}}\left[D^{\prime}(0)-i D^{\prime \prime}(0)\right], \\
& \left(S_{-}^{0}\right)^{2}=-\frac{\eta_{0}}{2 Y_{0}}\left[D^{\prime}(0)+i D^{\prime \prime}(0)\right] .
\end{aligned}
$$

From these equations we obtain four pairs of values for the $x, y$ components:

$$
\begin{aligned}
& S_{x}^{0}= \pm \frac{i}{2} \sqrt{\frac{\eta_{0}}{Y_{0}}}\left(\sqrt{D^{\prime}(0)+i D^{\prime \prime}(0)} \pm \sqrt{D^{\prime}(0)-i D^{\prime \prime}(0)}\right) . \\
& S_{y}^{0}= \pm \frac{1}{2} \sqrt{\frac{\eta_{0}}{Y_{0}}}\left(\sqrt{D^{\prime}(0)+i D^{\prime \prime}(0)} \mp \sqrt{D^{\prime}(0)-i D^{\prime \prime}(0)}\right) .
\end{aligned}
$$

(The double signs are correlated vertically but not horizontally.) Which solution-pair pertains to a given measurement cannot be determined from the equations alone. It would seem that ordinarily the overall plus-minus signs should be of no significance. The remaining sign-choice does affect the determination of the polarization index $\rho=S_{y}^{0} / S_{x}^{0}$. If we write $\rho^{(u)}$ and $\rho^{(l)}$ for the values associated with the upper and the lower signs, respectively, we find

$$
\rho^{(u)} \rho^{(l)}=-1 .
$$

With the aid of this equation a modicum of a priori information about the magnitude and/or the phase of $\rho$ should ordinarily be sufficient to resolve the ambiguity. For example, if the antenna is known to be approximately linearly polarized (on-axis) in a certain direction (we may choose the $y$ axis in that direction), then $|\rho|$ is distinctly greater than unity and one would choose $\rho^{(u)}$ or $\rho^{(l)}$ accordingly. However, if the polarization is nearly circular, the difference between the two indices becomes relatively small and the choice could be difficult.

The squared magnitude $\left|S_{x}^{0}\right|^{2}+\left|S_{y}^{0}\right|^{2}$, which determines the on-axis power gain, is unambiguous. In fact, for this gain we find

$$
G_{1}(0)=\frac{2 \pi k^{2}\left[\left|D^{\prime}(0)+i D^{\prime \prime}(0)\right|+\left|D^{\prime}(0)-i D^{\prime \prime}(0)\right|\right]}{1-\left|S_{00}\right|^{2}}
$$

(ii) Additional solutions permitted by symmetry. Certain types of symmetry enable one to determine certain off-axis values of the unknown functions. We consider one type of symmetry of frequent occurrence, exemplified by the fields of (a) rectangular waveguide open-ended or with a pyramidal horn, fed by the $T E_{10}$ mode in the waveguide; (b) circular waveguide open-ended or with a circularly symmetric horn, fed by the $T E_{11}$ mode in the waveguide; and (c) a transverse electric dipole. In these examples the symmetry may be analytically specified in terms of spectral components by

$$
\begin{aligned}
& S_{10 x}\left(-k_{x}, k_{y}\right)=S_{10 x}\left(k_{x},-k_{y}\right)=-S_{10 x}\left(k_{x}, k_{y}\right), \\
& S_{10 y}\left(-k_{x}, k_{y}\right)=S_{10 y}\left(k_{x},-k_{y}\right)=S_{10 y}\left(k_{x}, k_{y}\right) .
\end{aligned}
$$

(Here a specific orientation of structures and fields has of course been assumed.) We note in particular that $S_{10 x}$ must vanish on the coordinate axes. Hence, the four unknown functions involved in (5) are reduced to the single one, $S_{10 y}\left(k_{x}, 0\right)$ on the line $k_{y}=0$, and to the single func- 
tion $S_{10 y}\left(0, k_{y}\right)$ on the line $k_{x}=0$. From (5) we immediately obtain the separate equations determining these two functions,

$$
\begin{aligned}
& \eta_{2}\left[S_{10 y}\left(k_{x}, 0\right)\right]^{2}=\eta_{0} D^{\prime}\left(k_{x}, 0\right), \\
& \eta_{1}\left[S_{10 y}\left(0, k_{y}\right)\right]^{2}=\eta_{0} D^{\prime}\left(0, k_{y}\right) .
\end{aligned}
$$

Next we notice that on the diagonal line $k_{y}=k_{x}$, we have $S_{x}^{d} \equiv S_{x}$ and $S_{y}^{d} \equiv S_{y}$. Additionally, the symmetry furnishes $S_{x}=-S_{x}^{v}$ and $S_{y}=S_{y}^{v}$. Hence, of the six unknown functions involved in (5) and (9) essentially only two distinct ones survive,

$$
u_{x} \equiv S_{10 x}\left(k_{x}, k_{x}\right), \quad u_{y} \equiv S_{10 y}\left(k_{x}, k_{x}\right),
$$

say. Further, the two quadratics can easily be solved for these unknowns, as the following intermediate results show:

$$
\begin{gathered}
\eta_{2}\left(u_{x}-u_{y}\right)^{2}=\eta_{0}\left[D^{\prime}\left(k_{x}, k_{y}\right)+D^{\prime \prime}\left(k_{x}, k_{x}\right)\right], \\
\eta_{1}\left(u_{x}+u_{y}\right)^{2}=\eta_{0}\left[D^{\prime}\left(k_{x}, k_{x}\right)-D^{\prime \prime}\left(k_{x}, k_{x}\right)\right] .
\end{gathered}
$$

Hence in this case we can obtain solutions on the four lines, $k_{x}=0, k_{y}=0$, and $k_{y}= \pm k_{x}$.

\subsection{Generalized Three-Antenna Techniques}

In this subsection we discuss the analysis involved in techniques for determination of both power gain and polarization using three unknown (dissimilar) antennas. We require an antenna $T$, to be used only in transmitting; an antenna $R$, to be used only in reception; and an antenna $S$ to be used in both receiving and transmitting modes. We do not need to inquire whether either of the antennas $T$ and $R$ is reciprocal or even capable of operating in a "reversed" mode. We do require either that antenna $S$ be reciprocal or, if not reciprocal, capable of being "switched" to become its own adjoint $S^{a}$.

It is interesting and important that some kind of reciprocity ${ }^{12}$ is indispensably required a priori information. This requirement cannot be avoided by increasing the number of antennas involvedeven to the extent of using all possible combinations of $n$ transmitting and $m$ receiving antennas. But when the reciprocity requirement is met, as with a reciprocal or switchable antenna $S, 3$ antennas are sufficient. (Note that in the switchable case, $S$ and its adjoint do not coexist. Actual simultaneous possession of both $S$ and $S^{a}$ would distinctly change the character of the measurement problem: In this case one could use the generalized 2 -identical-antenna technique of the preceding subsection.)

Of course the use of 3 antennas in a measurement scheme is not in itself new. The use of 3 antennas in the roles of $T, R$, and $S$ ( $S$ being reciprocal) is recognizable in a discussion in Vol. 12 of the MIT Radiation Laboratory Series - material originating in the early 1940's [45]. However, in that discussion simplifying assumptions regarding polarization were made and the analytical problem was reduced to the use of scalar equations involving gain only. Analogous schemes using three electroacoustic transducers coupled by a fluid medium are well known [46]. The application of the requisite electroacoustic reciprocity relations was introduced by Maclean [47] and by Cook [48] in 1940 and 1941, respectively.

In this discussion we consider only on-axis values of antenna characteristics and refer to these quantities as the antenna characteristics. Determination of off-axis characteristics by three-

${ }^{12}$ Conceivably some linear relation, other than that provided by ordinary or adjoint reciprocity, between receiving and transmitting characteristics might be known a priori. This would not in general lead to the relatively simple equations that are obtained under the assumption made. 
antenna techniques has not yet been seriously considered, to our knowledge. (The corresponding electroacoustics problem, which is much simpler, has recently been completely solved under minimum assumptions in work as yet unpublished.)

When antenna polarization characteristics are fully taken into account, as in the present discussion, three-antenna techniques for the determination of on-axis characteristics require less a priori information than any other technique. Thus versions of the three-antenna technique have been the method of choice in several critical applications [e.g., 9,11].

The problem now posed is taken to be the determination of the receiving characteristic $\mathbf{R}_{01}(0)$ of the antenna $R$, the transmitting characteristic $\mathbf{T}_{10}(0)$ of the antenna $T$, and the four "right-side" characteristics $\mathbf{S}_{10}(0), \mathbf{S}_{01}(0), \mathbf{S}_{10}^{a}(0), \mathbf{S}_{01}^{\prime \prime}(0)$ of the antennas $S$ and $S^{a}$. We further choose to eliminate the receiving characteristics of $S$ and $S^{\prime \prime}$ by means of the reciprocity relations, which for $\mathbf{K}=0$ are simply

$$
\eta_{0} \mathbf{S}_{01}(0)=Y_{0} \mathbf{S}_{10}^{a}(0), \quad \eta_{0} \mathbf{S}_{01}^{a}(0)=Y_{0} \mathbf{S}_{10}(0)
$$

In this discussion we use the notation

$$
R_{x}=R_{02 x}(0), \quad S_{x}=S_{10 x}(0), \quad S_{x}^{a}=S_{10 x}^{a}(0), \quad T_{x}=T_{10 x}(0) \quad(x=x, y)
$$

for the components of the four remaining vector unknowns. Certainly, to determine these 8 (complex) quantities we need a system of 8 (complex) equations; the "core" of the problem, however, turns out to be the solution of six simultaneous quadratic equations for six of the unknowns. (This is the complete solution if antenna $S$ is reciprocal.) The remainder of the problem requires only the solution of two linear equations in two unknowns; this part will be called the "supplementary" problem.

The complete problem requires coupling-product data derived from transmission between antennas paired as shown in table 2. Transmission is from left to right (as already implied in the notation), and for each antenna-pair considered, the receiving antenna is used in two orientations differing by 90 degree rotation around the $\mathrm{z}$ axis. The first two columns in the table indicate two ways of utilizing the same data and are related by the interchange of the roles of $S$ and $S^{\prime \prime}$. If the antenna $S$ is reciprocal $\left(S=S^{a}\right)$, the first two columns coincide and reduce to the third; only the core problem remains. It will be sufficient to formulate and solve the algebraic problem represented in the first column of the table; the corresponding formulations and solutions may be obtained for the second column by interchanging $S$ and $S^{a}$ and for the case of reciprocal $S$ by eliminating the superscript " $a$ " (and with it the equations thus rendered superfluous).

TABle 2. Antenna pairings

\begin{tabular}{l|c|c|c}
\hline \hline \multirow{2}{*}{ For "core" problem: } & \multicolumn{2}{|c|}{$S \neq S^{\prime}$} & $S=S^{\prime \prime}$ \\
\cline { 2 - 4 } & $T \rightarrow R$ & $T \rightarrow R$ & $T \rightarrow R$ \\
& $S \rightarrow R$ & $S^{a} \rightarrow R$ & $S \rightarrow R$ \\
For "supplementary" problem: & $S^{a} \rightarrow R$ & $S \rightarrow R$ & - \\
\hline
\end{tabular}

For the transmission from $T$ to $R$, we have

$$
T_{x} R_{x}+T_{y} R_{y}=D_{R T}^{\prime}, \quad-T_{x} R_{y}+T_{y} R_{x}=D_{R T}^{\prime \prime} .
$$

These equations are an instance of (4.1-8) (except that here characteristics of both antennas are unknown). For the transmission from $S$ to $R$, we have

$$
S_{x} R_{x}+S_{y} R_{y}=D_{R S}^{\prime}, \quad-S_{x} R_{y}+S_{y} R_{x}=D_{R S}^{\prime \prime} .
$$


These equations are similarly an instance of (4.1-8). For the transmission from $T$ to $S^{a}$, we have

$$
-T_{x} S_{x}+T_{y} S_{y}=\eta_{0} D_{S T}^{\prime} / Y_{0}, \quad-T_{x} S_{y}-T_{y} S_{x}=\eta_{0} D_{S T}^{\prime \prime} / Y_{0}
$$

The receiving antenna, $S^{a}$, is here treated in the same way as was the receiving antenna in the twoantenna technique described in the preceding subsection (cf. (4.2-10)). This completes the formulation of the "core" problem. The solution is conveniently accomplished with the aid of circular polarization components, which are defined as in (4.1-9). Equations (3), (4), and (5) transform by pair to

$$
\left.\begin{array}{c}
T_{-} R_{-}=\frac{1}{2}\left(D_{R T}^{\prime}+i D_{R T}^{\prime \prime}\right) \equiv \Sigma_{R T}, \\
T_{+} R_{+}=\frac{1}{2}\left(D_{R T}^{\prime}-i D_{R T}^{\prime \prime}\right) \equiv \Delta_{R T} ;
\end{array}\right\}
$$

For the squares of the individual circular polarization components one finds without difficulty (but note that neither this set of equations nor the preceding sets can be generated by straightforward advancement of subscripts)

$$
\left.\begin{array}{l}
R_{-}^{2}=-\frac{Y_{0}}{\eta_{0}} \frac{\Sigma_{R T} \Sigma_{R S}}{\Sigma_{S T}},\left(T_{-} S_{-} \neq 0\right) \\
R_{+}^{2}=-\frac{Y_{0}}{\eta_{0}} \frac{\Delta_{R T} \Delta_{R S}}{\Delta_{S T}} ;\left(T_{+} S_{+} \neq 0\right)
\end{array}\right\}
$$

The inequalities shown in parentheses (which in effect prohibit circular polarizations) must be in force if indeterminacy in the respective associated equations is to be avoided. If circular polarizations do occur, then obviously many special cases are possible. For example, if the transmitting characteristic of antenna $S$ is (very nearly) right circularly polarized $\left(S_{y}=i S_{x}\right)$, then (very nearly) $S_{-}=0, \Sigma_{R S}=0, \Sigma_{S T}=0$, and $R_{-}$and $T_{-}$become experimentally indeterminate. However, the determination of the other circular components of $R$ and $T$, and the determination of the transmitting characteristics of antenna $S$ itself, would present no special difficulty. In practice, if a par- 
tial solution such as this is not sufficient, one might include additional antennas in the scheme. Antennas capable of being switched between right and left circular polarizations have been used for similar reasons, usually in less complicated circumstances.

It remains to dispose of what we called the supplementary problem earlier in this discussion. If antenna $S$ is reciprocal, there is no supplementary problem; if $S$ is not reciprocal, it remains to determine $\mathbf{S}_{10}^{a}(0)$. As suggested in the first column of table 2, this can be done by measurement with the receiving antenna $R$, for which calibration is presumptively available as a result of the core problem. Equations (4.1-11) are applicable; in notation adapted to the present context those equations become

$$
\begin{array}{r}
R_{+} S_{+}^{a}+R_{-} S_{-}^{a}=D_{R S}^{\prime}{ }^{a} \\
i R_{+} S_{+}^{a}-i R_{-} S_{-}^{a}=D_{R S}^{\prime \prime}{ }^{a} .
\end{array}
$$

Here complete solvability requires $R_{+} R_{-} \neq 0$.

Effective areas and power gains for antennas $R, S, S^{a}$, and $T$ (as pertinent) are readily expressed in terms of circular polarization components, which by reference to (4.1-9), are seen to be normalized so that

$$
\left|A_{x}\right|^{2}+\left|A_{y}\right|^{2}=\left|A_{+}\right|^{2}+\left|A_{-}\right|^{2} .
$$

Thus, for example, we obtain for antenna $S$ from (10) and (1.6-6)

$$
G_{1}(0)=\frac{4 \pi k^{2}}{1-\left|S_{00}\right|^{2}}\left(\left|\frac{\Delta_{R S} \Delta_{S T}}{\Delta_{R T}}\right|+\left|\frac{\sum_{R S} \Sigma_{S T}}{\sum_{R T}}\right|\right) .
$$

The corresponding effective area is given by (1.6-21a) or (1.6-21b), as appropriate.

Algebraic values for the individual linear polarization components may be obtained from (9), (10), or (11), as desired. For example, for antenna $S$

$$
\begin{aligned}
& S_{x}= \pm i \sqrt{\frac{\eta_{0}}{Y_{0}}}\left(\sqrt{\left.\frac{\sum_{R S} \Sigma_{S T}}{\sum_{R T}} \pm \sqrt{\frac{\Delta_{R S} \Delta_{S T}}{\Delta_{R T}}}\right),}\right. \\
& S_{y}= \pm \sqrt{\frac{\eta_{0}}{Y_{0}}}\left(\sqrt{\frac{\sum_{R S} \Sigma_{S T}}{\Sigma_{R T}}} \mp \sqrt{\frac{\Delta_{R S} \Delta_{S T}}{\Delta_{R T}}}\right),
\end{aligned}
$$

where the double signs are correlated vertically but not horizontally. As far as sign ambiguities are concerned, these equations have the same algebraic structure as (4.2-17) and the earlier discussion is again applicable.

\section{Appendices}

\subsection{Appendix A: Reciprocity Theorems}

The domain of the electromagnetic fields under consideration is the source-free region $\mathrm{V}$ bounded externally by the surfaces $F_{1}$ and $F_{2}$ and internally by the closed surface $S_{0}+S_{a}$, which encloses the source or detector associated with the antenna considered. (See sec. 1.1 and fig. 1.) A passive antenna is a scatterer (sometimes called a "loaded scatterer"); if the structure is merely a scatterer, the surface $S_{0}+S_{a}$ is irrelevant and may be disregarded.

We shall write Maxwell's equations in a form especially suited to the purposes of the present discussion. We associate with the given system, described by the constitutive equations (1.5-1), the "Maxwellian" operator 


$$
M=\left(\begin{array}{cc}
i \omega \boldsymbol{\epsilon} \cdot & i \omega \boldsymbol{\tau} \cdot \nabla \times \\
-i \omega \boldsymbol{\nu} \cdot \nabla \times & -i \omega \boldsymbol{\mu} \cdot
\end{array}\right)
$$

and place the vectors $\mathbf{E}$ and $\mathbf{H}$ in the column matrix

$$
\Phi=\left(\begin{array}{l}
\mathbf{E} \\
\mathbf{H}
\end{array}\right)
$$

so that Maxwell's equations for a possible field in the system are expressed by

$$
M \Phi=0 .
$$

Using the constitutive equations (1.5-2) we similarly obtain for the Maxwellian operator associated with the adjoint system

$$
\tilde{M}=\left(\begin{array}{cc}
i \omega \tilde{\boldsymbol{\epsilon}} \cdot & -i \omega \tilde{\boldsymbol{\nu}} \cdot+\nabla \times \\
i \omega \tilde{\boldsymbol{\tau}} \cdot+\nabla \times & -i \omega \tilde{\boldsymbol{\mu}} .
\end{array}\right)
$$

where $\tilde{M}$ is in a natural sense the transpose of the operator $M$. Maxwell's equations for the fields in the adjoint system take the form

$$
\tilde{M} \Phi=0
$$

The adjoint system represents a possible physical embodiment of the mathematical concept of adjoint differential expressions, which may be defined as follows. Let

$$
\Phi^{\prime}=\left(\begin{array}{l}
\mathbf{F}^{\prime} \\
\mathbf{G}^{\prime}
\end{array}\right), \quad \Phi^{\prime \prime}=\left(\begin{array}{c}
\mathbf{F}^{\prime \prime} \\
\mathbf{G}^{\prime \prime}
\end{array}\right)
$$

where $\mathbf{F}^{\prime}, \mathbf{G}^{\prime}, \mathbf{F}^{\prime \prime}$, and $\mathbf{G}^{\prime \prime}$ are arbitrary differentiable vector functions defined in $\mathrm{V}$. Then we can associate a unique adjoint differential expression $M^{a}$ with $M$ by requiring that

$$
\tilde{\Phi}^{\prime \prime} \cdot M \Phi^{\prime}-\tilde{\Phi}^{\prime} \cdot M^{a} \Phi^{\prime \prime} \equiv D
$$

where $D$ is a divergence expression, be an identity in $\Phi^{\prime}$ and $\Phi^{\prime \prime}$. This leads to

$$
M^{a}=\tilde{M}
$$

and

$$
D=\nabla \cdot\left(\mathbf{F}^{\prime} \times \mathbf{G}^{\prime \prime}-\mathbf{F}^{\prime \prime} \times \mathbf{G}^{\prime}\right)
$$

Thus the adjoint operator turns out to be the transpose. ${ }^{13}$

We may now easily obtain the basic theorem for our purposes. If we replace $\mathbf{F}^{\prime}, \mathbf{G}^{\prime}$ and $\mathbf{F}^{\prime \prime}, \mathbf{G}^{\prime \prime}$ by $\mathbf{E}^{\prime}, \mathbf{H}^{\prime}$ and $\mathbf{E}^{\prime \prime}, \mathbf{H}^{\prime \prime}$, which satisfy (1) and (2), respectively, we have

$$
\nabla \cdot\left(\mathbf{E}^{\prime} \times \mathbf{H}^{\prime \prime}-\mathbf{E}^{\prime \prime} \times \mathbf{H}^{\prime}\right)=0
$$

throughout the region $\mathrm{V}$. This is a generalization of the well-known Lorentz relation. It may very easily be modified to include the effects of both electric and magnetic current sources embedded in $\mathrm{V}$ [37]. Here, however, we are interested in excitation of the systems by means of incident waves

${ }^{13}$ To avoid possible confusion, we emphasize that the adjoint arising here is definable essentially by transposition (without complex conjugation); it is not the Hermitian adjoint that is frequently useful in other physical problems. For discussion of the theory of adjoint differential expressions see especially Lanczos [49] or (for ordinary differential equations) Courant-Hilbert [50]. Equation (5a) incidentally contains the nontrivial result that the operator $\nabla \times$ is self-adjoint. 
(in space or waveguide). With or without current-source terms, (6) characterizes what may properly be called adjoint reciprocity.

If the constitutive tensors obey the symmetry relations

$$
\boldsymbol{\epsilon}=\tilde{\boldsymbol{\epsilon}}, \quad \boldsymbol{\mu}=\tilde{\boldsymbol{\mu}}, \quad \text { and } \boldsymbol{\tau}=-\tilde{\boldsymbol{\nu}}
$$

then (as may be seen from $\left(1.5^{-1,-2))}\right.$ the adjoint system and the original system are identical (and $\left.M^{a}=\tilde{M}=M\right)$. In this case we say that the linear differential operators and the systems are selfadjoint-and ordinary reciprocity obtains. Equation 6 still holds; $\mathbf{E}^{\prime}, \mathbf{H}^{\prime}$ and $\mathbf{E}^{\prime \prime}, \mathbf{H}^{\prime \prime}$ may be interpreted as distinct electromagnetic fields in one and the same system.

To apply the generalized Lorentz relation, we first take the volume integral of the expression over the region $\mathrm{V}$ and use the divergence theorem. This yields

$$
\int_{S_{0}} \boldsymbol{L} \cdot \mathbf{n}_{0} d S=\sum_{q} \int_{F_{q}} \underline{\boldsymbol{L}} \cdot \mathbf{n}_{q} d \mathbf{R}
$$

where $\boldsymbol{L}=\mathbf{E}^{\prime} \times \mathbf{H}^{\prime \prime}-\mathbf{E}^{\prime \prime} \times \mathbf{H}^{\prime}$ and the unit normals are inward on $S_{0}$ and outward on $F_{q}$ (as prescribed in subsections 1.1 and 1.2). Next we substitute the modal representations of the fields $\mathbf{E}^{\prime}, \mathbf{H}^{\prime}$ and $\mathbf{E}^{\prime \prime}, \mathbf{H}^{\prime \prime}$ on $S_{0}, F_{1}$ and $F_{2}$, using single and double primes to distinguish the spectral variables associated with the respective fields. One obtains after some analysis the generalized reciprocity lemma

$\eta_{0}\left(a_{0}^{\prime \prime} b_{0}^{\prime}-a_{0}^{\prime} b_{0}^{\prime \prime}\right)$

$$
=\sum_{p} \int_{\mathbf{L}} \sum_{n}\left[a_{p}^{\prime \prime}(n,-\mathbf{L}) b_{p}^{\prime}(n, \mathbf{L})-a_{p}^{\prime}(n, \mathbf{L}) b_{p}^{\prime \prime}(n,-\mathbf{L})\right] \eta_{n}(L) d \mathbf{L} .
$$

From this lemma, by making suitable special choices of excitation, we shall obtain the desired set of relations between the elements of the scattering matrix of the original system and the corresponding elements of the scattering matrix of the adjoint system.

For immediate reference we write down the scattering equations for the original system

$$
\begin{gathered}
b_{0}^{\prime}=S_{00} a_{0}^{\prime}+\sum_{p} \int_{\mathbf{L}} \sum_{n} S_{o p}(n, \mathbf{L}) a_{p}^{\prime}(n, \mathbf{L}) d \mathbf{L}, \\
b_{q}^{\prime}(m, \mathbf{K})=S_{q 0}(m, \mathbf{K}) a_{0}^{\prime}+\sum_{p} \int_{\mathbf{L}} \sum_{n} S_{q p}(m, \mathbf{K} ; n, \mathbf{L}) a_{p}^{\prime}(n, \mathbf{L}) d \mathbf{L} .
\end{gathered}
$$

and those for the adjoint system

$$
\begin{gathered}
b_{0}^{\prime \prime}=S_{00}^{a} a_{0}^{\prime \prime}+\sum_{p} \int_{\mathbf{L}} \sum_{n} S_{0 p}^{a}(n, \mathbf{L}) a_{p}^{\prime \prime}(n, \mathbf{L}) d \mathbf{L}, \\
b_{q}^{\prime \prime}(m, \mathbf{K})=S_{q 0}^{a}(m, \mathbf{K}) a_{0}^{\prime \prime}+\sum_{p} \int_{\mathbf{L}} \sum_{n} S_{q p}^{a}(m, \mathbf{K} ; n, \mathbf{L}) a_{p}^{\prime \prime}(n, \mathbf{L}) d \mathbf{L} .
\end{gathered}
$$

The superscript " $a$ " distinguishes quantities characteristic of the a., int system. The above sets of equations are instances of (1.3-2); each set is defined relative to the same basis fields and reference surfaces.

To find the relationships between transmitting and receiving characteristics, we let $\mathbf{E}^{\prime}, \mathbf{H}^{\prime}$ 
and $\mathbf{E}^{\prime \prime}, \mathbf{H}^{\prime \prime}$ be the fields corresponding to excitation of the respective systems by the incident waves represented by the following set of spectral variables:

$$
\begin{aligned}
& a_{0}^{\prime}=1, \quad a_{p}^{\prime}(n, \mathbf{L}) \equiv 0, \\
& a_{0}^{\prime \prime}=0, \quad a_{p}^{\prime \prime}(n, \mathbf{L})=\delta_{p q} \delta_{n m} \delta(\mathbf{L}-\mathbf{K}) .
\end{aligned}
$$

From (9) we find

$$
-b_{0}^{\prime \prime} \eta_{0}=b_{q}^{\prime}(m,-\mathbf{K}) \eta_{m}(K)
$$

and from (10) and (11)

$$
b_{q}^{\prime}(m, \mathbf{K})=S_{q 0}(m, \mathbf{K}), \quad \mathrm{b}_{0}^{\prime \prime}=S_{0 q}^{a}(m, \mathbf{K}) .
$$

Hence [observing that $\left.\eta_{m}(-K)=\eta_{m}(K)\right]$ we obtain

$$
\eta_{0} S_{0 q}^{a}(m, \mathbf{K})=-\eta_{m}(K) S_{q 0}(m,-\mathbf{K}) .
$$

Similarly, by interchanging the patterns of excitation (or by interchanging the designations of the original and the adjoint system), we obtain

$$
\eta_{0} S_{0 q}(m, \mathbf{K})=-\eta_{m}(K) S_{q 0}^{a}(m,-\mathbf{K}) .
$$

To obtain the scattering reciprocity relations, we consider each system to be excited by an incident plane wave, as represented in the following scheme.

$$
\begin{array}{ll}
a_{0}^{\prime}=0, & a_{p}^{\prime}(n, \mathbf{L})=\delta_{p p^{\prime}} \delta_{n n^{\prime}} \delta\left(\mathbf{L}-\mathbf{L}^{\prime}\right) ; \\
a_{0}^{\prime \prime}=0, & a_{p}^{\prime \prime}(n, \mathbf{L})=\delta_{p p^{\prime \prime}} \delta_{n n^{\prime \prime}} \delta\left(\mathbf{L}-\mathbf{L}^{\prime \prime}\right) .
\end{array}
$$

From (9) we find

$$
b_{p^{\prime \prime}}^{\prime}\left(n^{\prime \prime},-\mathbf{L}^{\prime \prime}\right) \eta_{n^{\prime \prime}}\left(L^{\prime \prime}\right)=b_{p^{\prime}}^{\prime \prime}\left(n^{\prime},-\mathbf{L}^{\prime}\right) \eta_{n^{\prime}}\left(L^{\prime}\right)
$$

and from (10) and (11)

$$
b_{q}^{\prime}(m, \mathbf{K})=S_{q p^{\prime}}\left(m, \mathbf{K} ; n^{\prime}, \mathbf{L}^{\prime}\right), \quad b_{q}^{\prime \prime}(m, \mathbf{K})=S_{q p^{\prime \prime}}\left(m, \mathbf{K} ; n^{\prime \prime}, \mathbf{L}^{\prime \prime}\right) .
$$

Hence (after changing variables to get rid of the primes), we obtain the set of scattering reciprocity relations

$$
\eta_{m}(K) S_{p q}(m, \mathbf{K} ; n, \mathbf{L})=\eta_{n}(L) S_{q p}^{a}(n,-\mathbf{L} ; m,-\mathbf{K})
$$

In this case interchanging $S$ and $S^{a}$ yields no further information.

If the reader has followed through any one of the above exercises, he will have no trouble in showing

$$
S_{00}=S_{00}^{a} .
$$

If we introduce the reciprocity dyadic

$$
\boldsymbol{\eta}=\eta_{1} \boldsymbol{\kappa}_{1} \boldsymbol{\kappa}_{1}+\eta_{2} \boldsymbol{\kappa}_{2} \boldsymbol{\kappa}_{2}
$$


and use the definitions (1.3-3), then (for example) (12b) becomes

$$
\eta_{0} \mathbf{S}_{0 q}(\mathbf{K})=\boldsymbol{\eta} \cdot \mathbf{S}_{q 0}^{a}(-\mathbf{K})
$$

and (13) is summed up in

$$
\boldsymbol{\eta}(\mathbf{K}) \cdot \mathbf{S}_{p q}(\mathbf{K}, \mathbf{L})=\mathbf{S}_{q p}^{a}(-\mathbf{L},-\mathbf{K})^{T} \cdot \boldsymbol{\eta}(\mathbf{L})
$$

where $\boldsymbol{\eta}(\mathbf{K})$ denotes $\boldsymbol{\eta}$ as a function of $\mathbf{K}$ and the superscript " $T$ " denotes the transposed dyadic (obtained by transposing the elementary dyads involved). It is worth noting that the minus sign appearing between the members of (12b) does not appear in (16). The reciprocity dyadic is manifestly diagonal in the $\boldsymbol{\kappa}_{1}, \boldsymbol{\kappa}_{2}$ basis; $(4.2-3)$ shows what happens when $\boldsymbol{\eta}$ is presented in the $\mathbf{e}_{x}, \mathbf{e}_{y}$ basis.

The Lorentz reciprocity relation seems to have been the quite generally preferred basis for the derivation of transmission or coupling equations (similar, at least in function, to our (2-15)) $[19,21,25,26]$. There is, however, no good reason why reciprocity should be invoked for that purpose: receiving characteristics can be defined analytically and operationally, independent of transmitting characteristics (as is done in this paper). Nevertheless, it is of some interest that the generalized Lorentz relation (8), or, more conveniently in the plane-wave framework, the lemma (9), can be used to derive transmission equations without invoking (ordinary) reciprocity.

\subsection{Appendix B: Dissipative Characteristics of Media; Comparison for Mutually Adjoint Media}

The concept that a medium may be "lossy," "lossless," or even "gainy" at a given point is familiar. We first need to give the mathematical expression of these qualitative properties of media. Then it will be easy to show that these properties are point-wise identical for mutually adjoint media.

According to properties of the complex Poynting's vector, dissipation or power loss per unit volume, $q(\mathbf{r})$, is given by

$$
q(\mathbf{r})=-\frac{1}{2} \operatorname{Re} \nabla \cdot(\mathbf{E} \times \overline{\mathbf{H}})
$$

In the medium described by (1.5-1), Maxwell's equations require

$$
\begin{aligned}
& \nabla \times \mathbf{E}=i \omega(\boldsymbol{\mu} \cdot \mathbf{H}+\boldsymbol{\nu} \cdot \mathbf{E}), \\
& \nabla \times \mathbf{H}=-i \omega(\boldsymbol{\epsilon} \cdot \mathbf{E}+\boldsymbol{\tau} \cdot \mathbf{H}) .
\end{aligned}
$$

(Note that these equations are source-free in the sense that they are satisfied by $\mathbf{E} \equiv \mathbf{H} \equiv 0$.) Using a standard vector identity, we combine (1) and (2) to obtain

$$
q(\mathbf{r})=-\frac{1}{2} \operatorname{Re}\left\{i \omega\left[\overline{\mathbf{H}} \cdot \boldsymbol{\mu} \cdot \mathbf{H}-\mathbf{E} \cdot \overline{\boldsymbol{\epsilon}} \cdot \overline{\mathbf{E}}+\overline{\mathbf{H}} \cdot\left(\boldsymbol{\nu}-\boldsymbol{\tau}^{*}\right) \cdot \mathbf{E}\right]\right\},
$$

where the superscript "** denotes the Hermitian conjugate. For our purpose it is convenient to rearrange this expression to read

$$
q(\mathbf{r})=\frac{\omega}{4}[\overline{\mathbf{E}}-i \overline{\mathbf{H}}] \cdot\left(\begin{array}{cc}
i\left(\boldsymbol{\epsilon}^{*}-\boldsymbol{\epsilon}\right) & \boldsymbol{\nu}^{*}-\boldsymbol{\tau} \\
\boldsymbol{\nu}-\boldsymbol{\tau}^{*} & i\left(\boldsymbol{\mu}^{*}-\boldsymbol{\mu}\right)
\end{array}\right) \cdot\left(\begin{array}{c}
\mathbf{E} \\
i \mathbf{H}
\end{array}\right) .
$$


The dissipative properties of the medium are thus seen to be determined by properties of the Hermitian matrix

$$
\Lambda(\mathbf{r})=\left(\begin{array}{cc}
i\left(\boldsymbol{\epsilon}^{*}-\boldsymbol{\epsilon}\right) & \boldsymbol{\nu} *-\boldsymbol{\tau} \\
\boldsymbol{\nu}-\tau^{*} & i\left(\boldsymbol{\mu}^{*}-\boldsymbol{\mu}\right)
\end{array}\right)
$$

which we call the loss matrix for the medium. For the present purpose the characteristics of the medium at a given point are appropriately classified according to the values assumable by $q(\mathbf{r})$ as $\mathbf{E}$ and $\mathbf{H}$, considered independent and arbitrary, vary. All possibilities are listed and named in table B-1.

There is no well-established terminology precisely fitting the physical properties being discussed. Terms generally chosen, and indeed the term "loss matrix," are biased by the tacit assumption that a medium normally is lossy. Thus if the loss matrix happens to be the zero matrix, we would ordinarily say that the medium is lossless at the point in question. But the medium would also be gainless at the same point. Neither physical nor technological restrictions prevent the occurrence of negative energy loss, which might be called gain (cf. Brand [51]).

Mathematical criteria for deciding the value class of Hermitian matrices are given by Mirsky [52]. In the lossless case, which is physically important but not counted as defining a value class by Mirsky, the loss matrix must be the zero matrix; this in turn requires $\epsilon=\epsilon^{*}, \boldsymbol{\tau}=\boldsymbol{\nu}^{*}$, and $\boldsymbol{\mu}=\boldsymbol{\mu}^{*}$.

TABLE B-1. Classification of dissipative characteristics

\begin{tabular}{|c|c|c|c|}
\hline $\begin{array}{l}\text { Values assumed } \\
\text { by } q(\mathbf{r}) \\
\text { r fixed }\end{array}$ & $\begin{array}{l}\text { Value class of loss } \\
\text { matrix at point } \mathbf{r}\end{array}$ & \multicolumn{2}{|c|}{$\begin{array}{l}\text { Dissipative characteristics } \\
\text { of medium at point } \mathbf{r}\end{array}$} \\
\hline$>0$ & Positive definite & Unconditionally & \\
\hline$>0$ & Positive semidefinite & Conditionally & T IOssy or passive \\
\hline$\equiv 0$ & Zero & \multicolumn{2}{|c|}{ Lossless, gainless or neutral } \\
\hline$\leqslant 0$ & Negative semidefinite & Conditionally & \multirow{2}{*}{ gainy or active } \\
\hline$<0$ & Negative definite & Unconditionally & \\
\hline$\frac{\geq}{<} 0$ & Indefinite & Indefinite & \\
\hline
\end{tabular}

Note: In the semidefinite cases both the zero and the nonzero values are to be assumable, and in the indefinite case both positive and negative values are to be assumable.

The medium adjoint to that described by (1.5-1) is defined and described by (1.5-2), according to which the adjoint expressions can be obtained by the replacements $\boldsymbol{\epsilon} \rightarrow \tilde{\boldsymbol{\epsilon}}, \tau \rightarrow-\tilde{\boldsymbol{\nu}}, \boldsymbol{\nu} \rightarrow-\tilde{\boldsymbol{\tau}}$, and $\boldsymbol{\mu} \rightarrow \tilde{\boldsymbol{\mu}}$. For the loss matrix this yields

$$
\Lambda^{a}(\mathbf{r})=\tilde{\Lambda}(\mathbf{r})
$$

i.e., $\Lambda^{a}(\mathbf{r})$ is the transpose of $\Lambda(\mathbf{r})$. This relation is sufficient to insure that the corresponding loss functions, $q(\mathbf{r})$ and $q^{a}(\mathbf{r})$, belong to the same value class (this can be seen, e.g., from the criteria given in Mirsky). We may say that the dissipative characteristics of a medium and its adjoint are the same, point by point. Whatever distribution of characteristics, including regions of active media (as in some antennas), is realized in one system will be realized in the adjoint system.

\subsection{Appendix C: Two-Dimensional, Spatial Sampling or Interpolation Theorem}

A variety of two-dimensional sampling theorems may be found in the literature $[42,53]$. The methods of derivation often used are unnecessarily complicated for our purposes. For the 
convenience of the reader we sketch a simple derivation of the simple desired result.

With the abbreviation $\mathbf{S}_{02}^{\prime}(\mathbf{K}) \cdot \mathbf{S}_{10}(\mathbf{K}) \exp (i \gamma d)=f(\mathbf{K})$, the equation under discussion, $(3.1-1)$, becomes

$$
b_{0}^{\prime}(\mathbf{P})=a_{0} F^{\prime} \int f(\mathbf{K}) e^{i \mathbf{K} \cdot \mathbf{P}} d \mathbf{K} .
$$

We have already noted that the mathematical requirement that $f(\mathbf{K})$,be "band limited" may be fulfilled extraordinarily and almost arbitrarily well. Let us therefore assume that nonzero values of $f(\mathbf{K})$ occur only in a finite region $\mathrm{K}$ of wavenumber space. For simplicity, we take $\mathrm{K}$ to be rectangular, bounded by the lines $k_{x}= \pm k_{1}, k_{y}= \pm k_{2}$. Then (1) may be written

$$
b_{0}^{\prime}(\mathbf{P})=a_{0} F^{\prime} \int_{-k_{2}}^{k_{2}} \int_{-k_{1}}^{k_{1}} f(\mathbf{K}) e^{i \mathbf{K} \cdot \mathbf{P}} d k_{i_{x}} d k_{y} .
$$

Further, $f(\mathbf{K})$ presumptively can be represented in $\mathrm{K}$ by a double Fourier series, with periods $2 h_{i_{1}}$ and $2 h_{i_{2}}$ :

$$
f(\mathbf{K})=\sum_{r=-\infty}^{\infty} \sum_{s=-\infty}^{\infty} C_{r s} e^{-i \mathbf{K} \cdot \mathbf{P}_{r s}}
$$

where the coefficients are given by the usual formula

$$
C_{r s}=\frac{1}{4 k_{1} k_{i 2}} \int_{-k_{2}}^{k_{2}} \int_{-k_{1}}^{k_{11}} f(\mathbf{K}) e^{i \mathbf{K} \cdot \mathbf{P}_{r s}} d k_{x} d l_{i y}
$$

with

$$
\mathbf{P}_{r s}=\frac{r \pi}{l_{11}} \mathbf{e}_{x}+\frac{s \pi}{l_{i_{2}}} \mathbf{e}_{y}
$$

Comparing (4) and (2), we see that the $C_{r s}$ are proportional to $b_{0}^{\prime}(\mathbf{P})$ evaluated for $\mathbf{P}=\mathbf{P}_{r s}$, so that (3) may be written

$$
f(\mathbf{K})=\frac{1}{4 h_{11} l_{i 2} a_{0} F^{\prime}} \sum_{r, s} b_{0}^{\prime}\left(\mathbf{P}_{r s}\right) e^{-i \mathbf{K} \cdot \mathbf{P}_{r s}}
$$

Thus $f(\mathbf{K})$ is completely determined by the sampling of data at the discrete points $\mathbf{P}_{r s s}$. This is the result used in $(3.1-4)$.

The usual objective in sampling theory is the reconstruction of the sampled function. In the present instance this is easily accomplished by substituting (6) in (2) and integrating. The result is an expression involving cardinal functions and expressing $b_{0}^{\prime}(\mathbf{P})$ at all points in terms of its values at the lattice points.

One should observe the reciprocal relation between the size of the region $\mathrm{K}$ in wavenumber space and the size of the elementary cell in $x, y$ space. The smaller the former, the larger the latter-and the smaller the required density of sampling points in $x, y$ space.

\section{References}

[1] Kerns, D. M., and Dayhoff, E. S., Theory of diffraction in microwave interferometry, J. Res. Nat. Bur. Stand. (U.S.), 64B, (Math. and Math. Phys.) 1-13 (Jan.-Mar. 1960). 
[2] Kerns, D. M., Correction of near-field antenna measurements made with an arbitrary but known measuring antenna, Electron. Lett. 6, 346-347 (May 1970).

[3] Kerns, D. M., Scattering-matrix description and near-field measurements of electroacoustic transducers, J. Acoust. Soc. Am. 57, 497-507 (Feb. 1975).

[4] Cohen, M. H., Reciprocity theorem for anisotropic media, Proc. IRE (Correspondence) 43, 103 (Jan. 1955).

[5] Kerns, D. M., Generalized variational principles for electromagnetic vibrations; application to the theory of waveguide junctions. pp. 253-260, in Electromagnetic Theory and Antennas (Proc. 1962 Symp.. Copenhagen, Denmark, E. C. Jordan, Ed., Pergamon Press, London, 1963).

[6] Wacker, P. F., Theory and numerical techniques for accurate extrapolation of near-zone antenna and scattering measurements, NBS Report (unpublished) (April 1972).

[7] Kerns, D. M., Plane-wave spectra and spectral coupling products for arbitrary radiating and scattering systems, NBS Report (unpublished) (March 1972).

[8] Baird, R. C., Newell, A. C., Wacker, P. F., and Kerns, D. M., Recent experimental results in near-field antenna measurements, Electron. Lett. 6, 349-351 (May 1970).

[9] Newell. A. C., and Kerns, D. M., Determination of both polarisation and power gain of antennas by a generalised 3-antenna measurement method, Electron. Lett. 7, 68-70, (Feb. 11, 1971).

[10] Joy. E. B., and Paris, D. T., Spatial sampling and filtering in near-field measurements, IEEE Trans. Antennas Prop. AP-20, 253-261 (May 1972).

[11] Newell, A. C., Baird, R. C., and Wacker, P. F., Accurate measurement of antenna gain and polarization at reduced distances by an extrapolation technique. IEEE Trans. Antennas Prop. AP-2 1, 418-431 (1973).

[12] Kanda, M., Accuracy considerations in the measurement of the power gain of a large microwave antenna. IEEE Trans. Antennas Prop. AP-23, 407-411 (1975).

[13] Yaghjian, A. D., Upper-bound errors in far-field antenna parameters determined from planar near-field measurements, Part 1: Analysis, Nat. Bur. Stand. (U.S.), Tech. Note 667 (1975).

[14] Newell, A. C., Upper-bound errors in far-field antenna parameters determined from planar near-field measurements, Part 2: Analysis and computer simulation, Nat. Bur. Stand. (U.S.) Tech. Note 671 (1975).

[15] Johnson. R. C., Ecker. H. A., and Hollis, J. S., Determination of far-field antenna patterns from near-field measurements, Proc. IEEE 61, 1668-1694 (Dec. 1973).

[16] Woonton. G. A., On the measurement of diffraction fields, Proc. McGill Symp. Microwave Optics. pp. 347-350 (McGill University, Montreal, Quebec, Canada, 1953). B. S. Karasik, Ed., Air Force Cambridge Research Center, Cambridge, Mass. (1959).

[17] Dayhoff, E. S., Corrections in high accuracy Fresnel region microwave interferometry, Nat. Bur. Stand. (U. S.), Office of Basic Instrumentation Report (unpublished) (1956).

[18] Kerns, D. M., Wavelength correction in a microwave interferometer, URSI-IRE Meeting, Washington, D.C. (1957).

[19] Brown, J., A theoretical analysis of some errors in aerial measurements, Proc. Inst. Elec. Eng. 105C, 343-351 (Feb. 1958).

[20] Brown, J., A generalized form of the aerial reciprocity theorem, Proc. Inst. Elec. Eng. 105C, 472-475 (1958).

[21] Jull, E. V., An investigation of near-field radiation patterns measured with large antennas, IRE Trans. Antennas Prop. AP-10, 363-369 (1962).

[22] Jull, E. V., The estimation of aerial radiation patterns from limited near-field measurements, Proc. IEE 110,501-506 (1963).

[23] Brown, J., and Jull, E. V., The prediction of aerial radiation patterns from near-field measurements, Proc. Inst. Elec. Eng. 108B, 635-644 (Nov. 1961).

[24] Martin, W. W., Computation of antenna radiation pattern from near-field measurements, IEEE Trans. Antennas Prop. (Letter) AP-15, 316-318 (1967).

[25] Jensen, F., Electromagnetic near-field far-field correlations, Ph. D. Diss. (Technical University of Denmark, Lyngby, 1970).

[26] Leach, W. M., Jr., and Paris, D. T., Probe compensated near-field measurements on a cylinder, IEEE Trans. Antennas Prop. AP-2 1, 435-445 (1973).

[27] Wacker, P. F., Non-planar near-field measurements: spherical scanning, Nat. Bur. Stands. (U. S.), Report NBSIR 75-809 (1975). (Available as COM 75-10989 from the National Technical Information Service, Springfield, VA 22161.)

[28] Kerns, D. M., Analytical techniques for the correction of near-field antenna measurements made with an arbitrary but known measuring antenna, URSI-IRE Meeting, Washington, D.C. (April 29-May 2, 1963).

[29] Kerns, D. M., and Beatty, R. W., Basic Theory of Waveguide Junctions and Introductory Microwave Network Analysis (Pergamon, New York, 1967).

[30] Kerns, D. M., Matrix description of waveguide N-ports, NBS Report (unpublished) (1971).

[31] Kerns, D. M., Definitions of $\nu, i, Z, Y, a, b, \Gamma$, and $S$, Proc. IEEE 55, 892-900 (1967).

[32] Stratton, J. A., Electromagnetic Theory (McGraw-Hill, New York, 1941).

[33] Born, M., and Wolf, E., Principles of Optics (Pergamon Press, London, 1959).

[34] Kerns, D. M., Plane-wave scattering-matrix and generalized reciprocity relations for antennas and scatterers, NBS Report (unpublished) (1972).

[35] O'Dell T. H., Magnetoelectrics - a new class of materials, Electronics and Power, 266-267 (August 1965). 
[36] Condon, E. U., Theories of optical rotatory power, Rev. Mod. Phys. 9, 432-457 (Oct. 1937).

[37] Kong, Jin-Au and Cheng, D. K., Modified reciprocity theorem for bianisotropic media, Proc. IEE (London) 117, 349-350 (Feb. 1970).

[38] Harrington, R. F., and Villeneuve, A. T., Reciprocity relationships for gyrotropic media, IRE Trans. Microwave Theory Tech. MTT-6, 308-310 (July 1958).

[39] Beckmann, P., The Depolarization of Electromagnetic Waves (Golem Press, Boulder, Colorado, 1968).

[40] Hollis, J. S., Lyon, T. J., and Clayton, L., Jr., (Eds.), Microwave Antenna Measurements (Scientific Atlanta, Atlanta, Georgia, 1970).

[41] Kerns, D. M., Antenna measurements with arbitrary antennas at arbitrary distances, contribution in High-Frequency and Microwave Field-Strength Precision Measurement seminar, NBS Report (unpublished) (1966).

[42] Petersen, D. P., and Middleton, D., Sampling and reconstruction of wave-number-limited functions in $\mathrm{N}$-dimensional Euclidean spaces, Information and Control 5, 279-323 (1962).

[43] Kerns, D. M., Analysis of symmetrical waveguide junctions, J. Res. Nat. Bur. Stand. (U.S.) 46, $267-282$ (April 1951).

[44] Kerns, D. M., New method of gain measurement using two identical antennas, Electron. Lett. 6, 348-349 (May 1970).

[45] Silver, S. (Ed), Microwave Antenna Theory and Design (Mass. Inst. Tech. Rad. Lab. Series, vol. 12, McGraw-Hill, New York 1949).

[46] Bobber, R. J., Underwater Electroacoustic Measurements (Naval Res. Lab., Underwater Sound Reference Div., Orlando, Florida, 1970).

[47] MacLean, W. R., Absolute measurement of sound without a primary standard, J. Acoust. Soc. Am. 12, 140-146 (1940).

[48] Cook. R. K., Absolute pressure calibration of microphones, J. Acoust. Soc. Am. 12, 415-420 (1941).

[49] Lanczos, C., Linear Differential Operators (D. Van Nostrand Co., Princeton, N.J., 1961).

[50] Courant, R., and Hilbert, D., Methods of Mathematical Physics (Interscience, New York, 1953).

[51] Brand, H., Schaltungslehre Linearer Mikrowellennetze (S. Hirzel Verlag, Stuttgart, 1970).

[52] Mirsky, L., An Introduction to Linear Algebra (Oxford University Press, Oxford, 1955).

[53] Goodman. J. W.. Introduction to Fourier Optics (McGraw-Hill Book Company, New York, 1968).

[54] Newell, A. C., and Crawford, M. L., Planar near-field measurements on high performance array antennas, Nat. Bur. Stand. (U.S.), Report NBSIR 74-380 (July 1974). (Available as COM 74-11686 from the National Technical Information Service, Springfield, VA 22161.)

(Paper 80B1-431) 